\title{
Mathematical Modelling of the Inhibitory Role of Regulatory T Cells in Tumor Immune Response
}

\author{
Zhongtao Yang, ${ }^{1,2}$ Cuihong Yang, ${ }^{1}$ Yueping Dong $\mathbb{D}^{1},{ }^{1}$ and Yasuhiro Takeuchi ${ }^{3}$ \\ ${ }^{1}$ School of Mathematics and Statistics, Central China Normal University, Wuhan 430079, China \\ ${ }^{2}$ School of Finance and Mathematics, Huainan Normal University, Huainan 232038, China \\ ${ }^{3}$ College of Science and Engineering, Aoyama Gakuin University, Sagamihara 252-5258, Japan
}

Correspondence should be addressed to Yueping Dong; dongyueping0531@gmail.com

Received 15 May 2020; Accepted 3 July 2020; Published 12 August 2020

Guest Editor: Songbai Guo

Copyright (c) 2020 Zhongtao Yang et al. This is an open access article distributed under the Creative Commons Attribution License, which permits unrestricted use, distribution, and reproduction in any medium, provided the original work is properly cited.

\begin{abstract}
The immune system against tumors acts through a complex dynamical process showing a dual role. On the one hand, the immune system can activate some immune cells to kill tumor cells (TCs), such as cytotoxic T lymphocytes (CTLs) and natural killer cells (NKs), but on the other hand, more evidence shows that some immune cells can help tumor escape, such as regulatory T cells (Tregs). In this paper, we propose a tumor immune interaction model based on Tregs-mediated tumor immune escape mechanism. When helper T cells' (HTCs) stimulation rate by the presence of identified tumor antigens is below critical value, the coexistence (tumor and immune) equilibrium is always stable in its existence region. When HTCs stimulation rate is higher than the critical value, the inhibition rate of effector cells (ECs) by Tregs can destabilize the coexistence equilibrium and cause Hopf bifurcations and produce a limit cycle. This model shows that Tregs might play a crucial role in triggering the tumor immune escape. Furthermore, we introduce the adoptive cellular immunotherapy (ACI) and monoclonal antibody immunotherapy (MAI) as the treatment to boost the immune system to fight against tumors. The numerical results show that ACI can control TCs more, while MAI can delay the inhibitory effect of Tregs on ECs. The result also shows that the combination of both immunotherapies can control TCs and reduce the inhibitory effect of Tregs better than a single immunotherapy can control.
\end{abstract}

\section{Introduction}

Tumors can be benignant (not cancerous), premalignant (precancerous), and malignant (cancerous). Every year millions of people suffer with cancer and die from this disease throughout the world [1]. It is important to understand tumor's mechanisms of establishment and destruction, cell-mediate immunity with cytotoxic $\mathrm{T}$ lymphocytes (CTLs), and natural killer cells (NKs), generally called effector cells (ECs) that are cytotoxic to tumor cells (TCs), and play a basic role in immune response against tumors [2, 3]. Moreover, efficient antitumor immunity requires the action of helper T cells (HTCs), which can directly activate naive $\mathrm{CD} 8^{+} \mathrm{T}$ cells to differentiate into CTLs [4-6]. Recently, it has been reported that regulatory T cells (Tregs) can inhibit CTLs and promote the escape of TCs [7]. Tregs suppress immune cells, and when the war between T cells and infection is over, the Tregs signal to stop [8]. Cancer immunotherapy fights against cancer by strengthening the body's immune system, but the involvement of Tregs inhibits the immune response and turns off the anticancer effect. Tregs inhibition is important in the dynamics of the tumor immune system, which is one motivation of this work.

Adoptive $\mathrm{T}$ cell immunotherapy (ACI) as a common immunotherapy involves injecting adoptive $\mathrm{T}$ cells directly into tumor patients [9-11]. Its advantages are good destruction of tumor and persistence, while its disadvantages are serious toxic and side effects. The monoclonal antibody immunotherapy (MAI) is the immune checkpoint inhibitor $[12,13]$, which has the advantage of removing the suppression state of the immune system and restoring the immune function of the body to TCs and the disadvantage of having serious immune-related adverse reactions. 
Tregs have become an important target in tumor immunotherapy because of their contribution to tumor immune escape. Cytotoxic T lymphocyte antigen 4 (CTLA-4) is a marker that is expressed on the surface of activated T cells and transmits inhibitory signals in the immune response [14-16]. Blocking CTLA-4 can reduce the inhibitory activity of Tregs, and the anti-CTLA-4 humanized monoclonal antibody Ipilimumab and Tremelimumab are used to treat advanced melanoma and malignant mesothelioma, respectively [17]. Similar to CTLA-4, programmed death receptor 1 (PD-1) can also promote the activation and development of Tregs [18-21]. Blocking PD-1 can prevent the development of Tregs and prevent the conversion of HTCs into Tregs [22]. Currently, OPDIVO (Nivolumab), an anti-PD-1 monoclonal antibody, has been approved by the US FDA for the treatment of melanoma, renal cell carcinoma, and non-small cell lung cancer [23-25]. Establishment of a mathematical model to study the immunotherapy on the reduction of Tregs inhibition has both theoretical and practical significance.

In order to describe the mechanisms of host's own immune response to against TCs, various types of mathematical models have been proposed [26-43]. The modelling of the tumor immune system described by ordinary differential equations (ODEs) has a long history, which can be traced back to the classic research of Stepanova in 1980 [26]. In 1994, Kuznetsov et al. established the famous two-dimensional ODEs model, postulating that tumor growth follows the Logistic growth pattern. They evaluated the parameters of the model by fitting experimental data from mice [27]. In 2003, Stolongo-Costa et al. assumed that TCs follows the exponential growth pattern and constructed a two-dimensional ODEs model. They analyzed the basic properties of the model and provided conditions for stability of the tumor-free equilibrium, explaining its epidemiological significance [28]. In 2004, Galach simplified Kuznetsov's system to account for the effect of immune delay on the tumor immune system [29]. In 2014, Dong et al. constructed a three-dimensional ODEs model focusing on the effects of HTCs on the tumor immune system [4].

In 1998, Kirschner and Panetta generalized Kuznetsov-Taylor model and illustrated the dynamics between TCs, ECs, and IL-2. They firstly introduced ACI into their model which can explain both short-term tumor oscillations in tumor sizes as well as long-term tumor relapse [11]. In 2003 , in order to study the role of cytokine therapy in the activation of the immune system, Stolongo-Costa et al. introduced cycle therapy term $F \cos ^{2} \omega t$ and established a cycle immunotherapy model. They obtained some thresholds of the frequency and intensity of immunotherapy [28]. In 2006, de Pillis et al. constructed the six-dimensional ODEs model to investigate the effects of combined chemotherapy and immunotherapy on tumor control. They briefly analyzed the nature of the model and discussed the optimal treatment using optimal control theory [30]. In 2008, Bunimovich-Mendrazitsky et al. established a pulsed differential equation model with Bacillus Calmette-Guerin tumor immunotherapy. They obtained the critical threshold and pulse frequency of BCG injection dose that could successfully treat superficial bladder cancer [31]. In 2012, Wilson and Levy established a mathematical model containing Tregs. They studied the absence of treatment, vaccine treatment, anti-TGF treatment, and combination vaccine and anti-TGF treatment, as well as sensitivity analysis of some important parameters [8]. In 2018, Radunskaya et al. established a mathematical model with blood, spleen, and tumor compartments to study PD-L1 inhibitors in the role of tumor immunotherapy. The model was used to fit parameters with the experimental data. The results showed that increasing the resistance of $\mathrm{PD}-\mathrm{L} 1$ doses can greatly improve the clearance rate of tumor [32].

This paper investigates the role of Tregs in the tumor immune system. Therefore, we incorporate the fourth population of Tregs into the previous system in [8]. For the mathematical simplicity, a bilinear term also has been used to describe the interactions between immune response and tumor. To our knowledge, HTCs can recognize TCs and promote the growth of ECs. And ECs can provide direct protective immunity by attacking TCs. When there are more HTCs and ECs, in order to maintain immune homeostasis, the body will produce corresponding Tregs to suppress ECs, and Tregs originating from both HTCs and ECs. Then, we establish a four-dimensional ODEs model described as below:

$$
\left\{\begin{array}{l}
\frac{d T(t)}{d t}=a T(t)(1-b T(t))-n E(t) T(t), \\
\frac{d E(t)}{d t}=p E(t) H(t)-q R(t) E(t)-d_{1} E(t), \\
\frac{d H(t)}{d t}=s_{2}+k_{2} T(t) H(t)-d_{2} H(t), \\
\frac{d R(t)}{d t}=r_{1} E(t)+r_{2} H(t)-d_{3} R(t),
\end{array}\right.
$$

where $T(t), E(t), H(t)$, and $R(t)$ represent the populations of TCs, ECs, HTCs, and Tregs, respectively. The first equation describes the rate change of TCs population. The tumor follows logistic growth dynamics with growth rate $a$, and the maximum capacity is $1 / b . n$ represents the loss rate of TCs by ECs interaction. The second equation describes the rate change of the ECs population. $d_{1}$ is the mortality rate of ECs. $p$ is the activation rate of ECs by HTCs, and $q$ is the inhibition rate of Tregs on ECs. The third equation describes the rate change of the HTCs population, $s_{2}$ is birth rate of HTCs produced in the bone marrow, and HTCs have a natural lifespan of an average $1 / d_{2}$ days. $k_{2}$ is HTCs stimulation rate by the presence of identified tumor antigens. The fourth equation gives the rate change of the Tregs population, $r_{1}$ and $r_{2}$ are the activation rates of Tregs by ECs and HTCs, respectively. $d_{3}$ represents per capita decay rate of Tregs. A diagram of the various interactions between these cell populations is shown in Figure 1.

We nondimensionalize model (1) by taking the following scaling: 


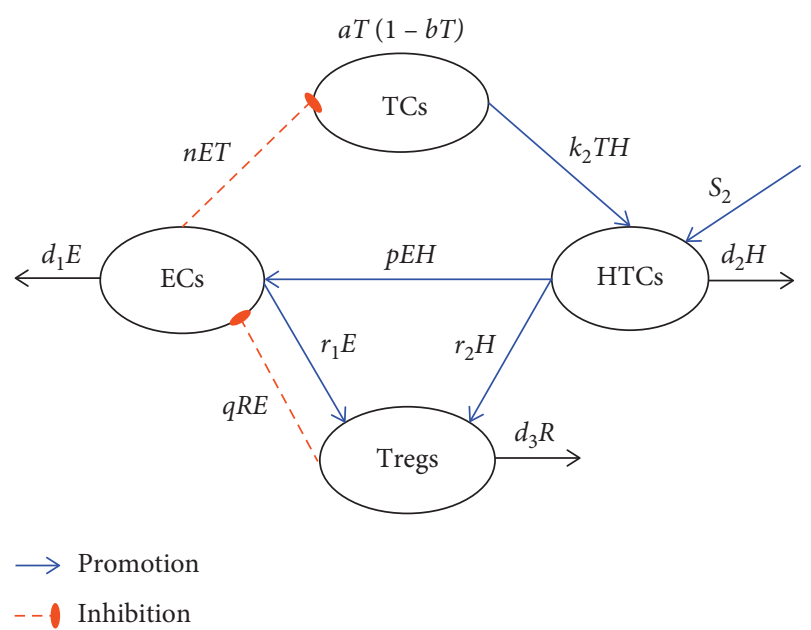

Figure 1: A diagram of interactions among the different cell populations in model (1).

$$
\begin{aligned}
t & =\frac{\tau}{n T_{0}}, T(t)=T_{0} x(\tau), E(t)=E_{0} y(\tau), H(t) \\
& =H_{0} z(\tau), R(t)=R_{0} u(\tau), \alpha=\frac{a}{n T_{0}}, \beta=b T_{0}, \\
\rho & =\frac{p}{n}, \omega_{2}=\frac{k_{2}}{n}, \theta=\frac{q R_{0}}{n T_{0}}, \delta_{1}=\frac{d_{1}}{n T_{0}}, \delta_{2} \\
& =\frac{d_{2}}{n T_{0}}, \delta_{3}=\frac{d_{3}}{n T_{0}}, \sigma_{2}=\frac{s_{2}}{n T_{0} H_{0}}, \gamma_{1}=\frac{r_{1}}{n R_{0}}, \gamma_{2}=\frac{r_{2}}{n R_{0}},
\end{aligned}
$$

and we choose the scaling $T_{0}=E_{0}=H_{0}=R_{0}=10^{6}$. By replacing $\tau$ by $t$, we obtain the following scaled model:

$$
\left\{\begin{array}{l}
\frac{d x(t)}{d t}=\alpha x(t)(1-\beta x(t))-x(t) y(t), \\
\frac{d y(t)}{d t}=\rho y(t) z(t)-\theta y(t) u(t)-\delta_{1} y(t), \\
\frac{d z(t)}{d t}=\sigma_{2}+\omega_{2} x(t) z(t)-\delta_{2} z(t), \\
\frac{d u(t)}{d t}=\gamma_{1} y(t)+\gamma_{2} z(t)-\delta_{3} u(t),
\end{array}\right.
$$

with initial conditions

$$
\begin{aligned}
& x(0)=x_{0} \geq 0, \\
& y(0)=y_{0} \geq 0, \\
& z(0)=z_{0} \geq 0, \\
& u(0)=u_{0} \geq 0 .
\end{aligned}
$$

Here, $x, y, z$, and $u$ denote the dimensionless densities of TCs, ECs, HTCs, and Tregs populations, respectively.

\section{Model Analysis}

2.1. Well Posedness of Model (3). The following proposition establishes the well posedness of model (3) with initial conditions (4).

Proposition 1. The solutions $(x(t), y(t), z(t), u(t))$ of model (3) with initial conditions (4) are existent, unique, and nonnegative on the interval $[0,+\infty)$.

Proof. Since the right-hand side of model (3) is completely continuous and locally Lipschitz on the interval $[0,+\infty)$, there exists a constant $\delta>0$ such that the solutions $(x(t), y(t), z(t), u(t))$ of model (3) with initial conditions (4) are existent and unique on the interval $[0, \delta)$, where $0<\delta \leq+\infty[44]$.

From model (3) with initial conditions (4), we obtain

$$
\begin{aligned}
& x(t)=x_{0} e^{\int_{0}^{t}(\alpha(1-\beta(s))-y(s)) d s} \geq 0, \\
& y(t)=y_{0} e^{\int_{0}^{t}\left(\rho z(s)-\theta u(s)-\delta_{1}\right) d s} \geq 0, \\
& z(t)=z_{0} e^{\int_{0}^{t}\left(\omega_{2} x(\tau)-\delta_{2}\right) d \tau}+\sigma_{2} \int_{0}^{t} e^{\int_{s}^{t}\left(\omega_{2} x(\tau)-\delta_{2}\right) d \tau} d s \geq 0, \\
& u(t)=u_{0} e^{-\delta_{3} t}+\int_{0}^{t}\left(\gamma_{1} y(s)+\gamma_{2} z(s)\right) e^{-\delta_{3}(t-s)} d s \geq 0 .
\end{aligned}
$$

It is obvious to see that the solutions $(x(t), y(t), z(t), u(t))$ of model (3) with initial conditions (4) are nonnegative for all $t \geq 0$. The proof is complete.

2.2. Existence of Equilibria. In this section, we will study the existence of various equilibria for system (3). We set $(d x / d t)=0,(d y / d t)=0,(d z / d t)=0$, and $(d u / d t)=0$ in system (3), and we have

$$
\left\{\begin{array}{l}
\alpha x(1-\beta x)-x y=0 \\
\rho y z-\theta y u-\delta_{1} y=0 \\
\sigma_{2}+\omega_{2} x z-\delta_{2} z=0 \\
\gamma_{1} y+\gamma_{2} z-\delta_{3} u=0
\end{array}\right.
$$

Putting $x=0$ and $y=0$ yields the tumor-free and ECsfree equilibrium, namely,

$$
P_{0}=\left(x_{0}, y_{0}, z_{0}, u_{0}\right)=\left(0,0, \frac{\sigma_{2}}{\delta_{2}}, \frac{\gamma_{2} \sigma_{2}}{\delta_{2} \delta_{3}}\right),
$$

which always exists.

Putting $x=0$ and $y \neq 0$ yields the tumor-free equilibrium, namely,

$P_{1}=\left(x_{1}, y_{1}, z_{1}, u_{1}\right)=\left(0, \frac{\delta_{3}\left(\rho \sigma_{2}-\delta_{1} \delta_{2}\right)-\gamma_{2} \theta \sigma_{2}}{\theta \gamma_{1} \delta_{2}}, \frac{\sigma_{2}}{\delta_{2}}, \frac{\rho \sigma_{2}-\delta_{1} \delta_{2}}{\delta_{2} \theta}\right)$, 
which exists when $\rho>\delta_{1} \delta_{2} / \sigma_{2}$ and $\theta<\delta_{3}\left(\rho \sigma_{2}-\right.$ $\left.\delta_{1} \delta_{2}\right) / \gamma_{2} \sigma_{2} \triangleq \theta_{1}$.

Putting $x=1 / \beta$ yields the tumor-dominant equilibrium, namely,

$$
P_{2}=\left(x_{2}, y_{2}, z_{2}, u_{2}\right)=\left(\frac{1}{\beta}, 0, \frac{\beta \sigma_{2}}{\beta \delta_{2}-\omega_{2}}, \frac{\beta \gamma_{2} \sigma_{2}}{\delta_{3}\left(\beta \delta_{2}-\omega_{2}\right)}\right),
$$

which exists when $\omega_{2}<\beta \delta_{2} \triangleq \omega_{2}^{*}$.

Putting $x \neq 0$ and $x \neq 1 / \beta$ and eliminating $y, z$, and $u$ in (6), we have

$$
F(x)=A x^{2}+B x+C=0,
$$

where $A=-\alpha \beta \theta \gamma_{1} \omega_{2}<0, B=\theta \alpha \gamma_{1}\left(\omega_{2}^{*}+\omega_{2}\right)+\delta_{1} \delta_{3} \omega_{2}>0$, and $C=\delta_{3}\left(\rho \sigma_{2}-\delta_{1} \delta_{2}\right)-\theta\left(\alpha \gamma_{1} \delta_{2}+\gamma_{2} \sigma_{2}\right)$.

Then, we have the coexistence equilibrium

$$
\begin{aligned}
P^{*}= & \left(x^{*}, y^{*}, z^{*}, u^{*}\right)=\left(x^{*}, \alpha\left(1-\beta x^{*}\right), \frac{\sigma_{2}}{\delta_{2}-\omega_{2} x^{*}},\right. \\
& \left.\cdot \frac{\alpha \gamma_{1}\left(1-\beta x^{*}\right)\left(\delta_{2}-\omega_{2} x^{*}\right)+\gamma_{2} \sigma_{2}}{\delta_{3}\left(\delta_{2}-\omega_{2} x^{*}\right)}\right),
\end{aligned}
$$

which exists when $x^{*}<\mu \triangleq \min \left\{(1 / \beta),\left(\delta_{2} / \omega_{2}\right)\right\}$, where $x^{*}$ is the positive root of (10).

Below, we consider the existence condition of $P^{*}$ :

Case (1): $C \geq 0$, i.e., $\theta \leq \delta_{3}\left(\rho \sigma_{2}-\delta_{1} \delta_{2}\right) / \alpha \gamma_{1} \delta_{2}+\gamma_{2} \sigma_{2} \triangleq \theta_{0}$. Since $F(1 / \beta)=\theta \alpha \beta \gamma_{1} \delta_{2}+\delta_{1} \delta_{3} \omega_{2}+C \beta / \beta>0$ and $F\left(\delta_{2} /\right.$ $\left.\omega_{2}\right)=\theta \alpha \gamma_{1} \delta_{2}+\delta_{1} \delta_{2} \delta_{3}+C>0$, we have $1 / \beta<x^{*}$ and $\delta_{2} / \omega_{2}<x^{*}$, which contradicts with $x^{*}<\mu$. Hence, $P^{*}$ does not exist in this case.

Case (2): $C<0$, i.e., $\theta>\theta_{0}$.
Let $\Delta=B^{2}-4 A C=4 \alpha \beta \gamma_{1} \theta \omega_{2}\left(\delta_{3}\left(\rho \sigma_{2}-\delta_{1} \delta_{2}\right)-\theta\left(\alpha \gamma_{1} \delta_{2}+\right.\right.$ $\left.\left.\gamma_{2} \sigma_{2}\right)\right)+\left(\theta \alpha \gamma_{1}\left(\omega_{2}^{*}+\omega_{2}\right)+\delta_{1} \delta_{3} \omega_{2}\right)^{2}=\left[\theta \alpha \gamma_{1}\left(\omega_{2}-\omega_{2}^{*}\right)+\delta_{1}\right.$ $\left.\delta_{3} \omega_{2}\right]^{2}+4 \alpha \beta \theta \gamma_{1} \omega_{2} \sigma_{2}\left(\rho \delta_{3}-\theta \gamma_{2}\right)$.

If $\Delta<0$, then (10) does not have a positive root. Hence, $P^{*}$ does not exist in this case.

If $\Delta=0$, then (10) has one positive root $x^{*}$, where $x^{*}=$ $\theta \alpha \gamma_{1}\left(\omega_{2}^{*}+\omega_{2}\right)+\delta_{1} \delta_{3} \omega_{2} / 2 \alpha \beta \theta \gamma_{1} \omega_{2}>\omega_{2}^{*}+\omega_{2} / 2 \beta \omega_{2}=1 / 2\left(\left(\delta_{2} /\right.\right.$ $\left.\left.\omega_{2}\right)+(1 / \beta)\right) \geq \mu$, which contradicts with $x^{*}<\mu$. Hence, $P^{*}$ does not exist in this case.

If $\Delta>0$, then (10) has two positive roots, where $x_{+}^{*}=$ $\theta \alpha \gamma_{1}\left(\omega_{2}^{*}+\omega_{2}\right)+\delta_{1} \delta_{3} \omega_{2}+\sqrt{\Delta} / 2 \alpha \beta \theta \gamma_{1} \omega_{2}$ and $x_{-}^{*}=\theta \alpha \gamma_{1}$ $\left(\omega_{2}^{*}+\omega_{2}\right)+\delta_{1} \delta_{3} \omega_{2}-\sqrt{\Delta} / 2 \alpha \beta \theta \gamma_{1} \omega_{2}$. Since $x_{+}^{*}>\theta \alpha \gamma_{1}\left(\omega_{2}^{*}+\right.$ $\left.\omega_{2}\right)+\delta_{1} \delta_{3} \omega_{2} / 2 \alpha \beta \theta \gamma_{1} \omega_{2}>\omega_{2}^{*}+\omega_{2} / 2 \beta \omega_{2}=(1 / 2)\left(\left(\delta_{2} / \omega_{2}\right)+(1 /\right.$ $\beta)) \geq \mu$, which contradicts with $x^{*}<\mu$. If $x_{-}^{*}<\mu$, i.e., $F(1 / \beta)=$ $\left(\delta_{1} \delta_{3} \omega_{2}+\beta \delta_{2} \quad\left(\rho \sigma_{2}-\delta_{1} \delta_{2}\right)-\theta \beta \gamma_{2} \sigma_{2} / \beta\right)>0 \Rightarrow \theta<\left(\delta_{1} \delta_{3} \omega_{2}+\right.$ $\left.\beta \delta_{3}\left(\rho \sigma_{2}-\delta_{1} \delta_{2}\right) / \beta \gamma_{2} \sigma_{2}\right) \triangleq \theta_{2}$ and $F\left(\delta_{2} / \omega_{2}\right)=\sigma_{2}\left(\rho \delta_{3}-\theta \gamma_{2}\right)>$ $0 \Rightarrow \theta<\rho \delta_{3} / \gamma_{2} \triangleq \theta_{3}$, there exists a unique coexistence equilibrium $\quad P^{*}=\left(x^{*}, y^{*}, z^{*} \quad, u^{*}\right)=\left(x^{*}, \alpha\left(1-\beta x^{*}\right), \sigma_{2} /\right.$ $\left.\delta_{2}-\omega_{2} x^{*}, \quad \alpha \gamma_{1}\left(1-\beta x^{*}\right)\left(\delta_{2}-\omega_{2} x^{*}\right)+\gamma_{2} \sigma_{2} / \delta_{3}\left(\delta_{2}-\omega_{2} x^{*}\right)\right)$, where $x^{*}=x_{-}^{*}$.

The existence conditions for each equilibrium are given in Table 1.

2.3. Stability of Equilibria. In order to investigate the local stability of the above equilibria $P_{0}, P_{1}, P_{2}$, and $P^{*}$ of system (3), we linearize the system and obtain Jacobian matrix at each equilibrium $\bar{P}(\bar{x}, \bar{y}, \bar{z}, \bar{u})$ :

$$
\mathbf{J}(\bar{P})=\left(\begin{array}{cccc}
\alpha-2 \alpha \beta \bar{x}-\bar{y} & -\bar{x} & 0 & 0 \\
0 & \rho \bar{z}-\theta \bar{u}-\delta_{1} & \rho \bar{y} & -\theta \bar{y} \\
\omega_{2} \bar{z} & 0 & \omega_{2} \bar{x}-\delta_{2} & 0 \\
0 & \gamma_{1} & \gamma_{2} & -\delta_{3}
\end{array}\right) .
$$

The corresponding characteristic equation is

$$
\operatorname{det}(\mathbf{J}(\bar{P})-\lambda \mathbf{I})=\left|\begin{array}{cccc}
\alpha-2 \alpha \beta \bar{x}-\bar{y}-\lambda & -\bar{x} & 0 & 0 \\
0 & \rho \bar{z}-\theta \bar{u}-\delta_{1}-\lambda & \rho \bar{y} & -\theta \bar{y} \\
\omega_{2} \bar{z} & 0 & \omega_{2} \bar{x}-\delta_{2}-\lambda & 0 \\
0 & \gamma_{1} & \gamma_{2} & -\delta_{3}-\lambda
\end{array}\right|=0 .
$$

Theorem 1. System (3) always has one tumor-free and ECsfree equilibrium $P_{0}$, which is unstable.

Proof. At $P_{0}$, characteristic (13) becomes

$$
(\lambda-\alpha)\left(\lambda+\delta_{2}\right)\left(\lambda+\delta_{3}\right)\left(\lambda+\frac{\theta \gamma_{2} \sigma_{2}+\delta_{1} \delta_{2} \delta_{3}-\rho \sigma_{2} \delta_{3}}{\delta_{2} \delta_{3}}\right)=0
$$

Theorem 2. System (3) has one tumor-free equilibrium $P_{1}$, when $\rho>\delta_{1} \delta_{2} / \sigma_{2}$ and $\theta<\theta_{1}$, which is locally asymptotically stable (LAS) if the inequality $\theta<\theta_{0}$ holds.

Proof. At $P_{1}$, characteristic (13) becomes

$$
\begin{gathered}
\left(\lambda-\frac{\theta\left(\alpha \gamma_{1} \delta_{2}+\gamma_{2} \sigma_{2}\right)-\delta_{3}\left(\rho \sigma_{2}-\delta_{1} \delta_{2}\right)}{\theta \gamma_{1} \delta_{2}}\right) \\
\left(\lambda+\delta_{2}\right)\left(\lambda^{2}+\delta_{3} \lambda+\theta \gamma_{1} y_{1}\right)=0 .
\end{gathered}
$$
is unstable. 
TABLE 1: The existence and stability conditions of each equilibrium.

\begin{tabular}{ccc}
\hline & Existence conditions & Stability conditions \\
\hline$P_{0}$ & Always & Unstable \\
$P_{1}$ & $\theta<\theta_{1}$ & $\theta<\theta_{0}$ \\
$P_{2}$ & $\omega_{2}<\omega_{2}^{*}$ & $\theta>\theta_{2}$ \\
$P^{*}$ & $\theta>\theta_{0}\left\{\begin{array}{c}(i): \omega_{2} \leq \omega_{2}^{*}, \theta<\theta_{2}<\theta_{3} \\
(i): \omega_{2}>\omega_{2}^{*}, \theta<\theta_{3}<\theta_{2}\end{array}\right.$ & $(20)$ \\
\hline
\end{tabular}

Then, one root of characteristic equation is $-\delta_{2}<0$. It is easily noted that as $\delta_{3}>0, \theta \gamma_{1} y_{1}>0$, so $\lambda^{2}+\delta_{3} \lambda+\theta \gamma_{1} y_{1}=0$ has solutions with negative real parts. Therefore, $P_{1}$ is LAS when $\theta\left(\alpha \gamma_{1} \delta_{2}+\gamma_{2} \sigma_{2}\right)-\delta_{3}\left(\rho \sigma_{2}-\delta_{1} \delta_{2}\right) / \theta \gamma_{1} \delta_{2}<0$, that is, $\theta<\theta_{0}$.

Theorem 3. System (3) has one tumor-dominant equilibrium $P_{2}$, when $\omega_{2}<\omega_{2}^{*}$, which is LAS if the inequality $\theta>\beta \delta_{3}\left(\rho \sigma_{2}-\delta_{1} \delta_{2}\right)+\delta_{1} \delta_{3} \omega_{2} / \beta \gamma_{2} \sigma_{2} \equiv \theta_{2}$ holds.

Proof. At $P_{2}$, characteristic (13) becomes

$$
(\lambda+\alpha)\left(\lambda+\delta_{3}\right)\left(\lambda-\frac{\omega_{2}-\omega_{2}^{*}}{\beta}\right)\left[\lambda-\left(\rho z_{2}-\theta u_{2}-\delta_{1}\right)\right]=0 .
$$

The three roots of characteristic equation are $\lambda_{1}=-\alpha<0, \lambda_{2}=-\delta_{3}<0$, and $\lambda_{3}=\omega_{2}-\omega_{2}^{*} / \beta<0, \quad$ if $\omega_{2}<\omega_{2}^{*}$. So, $P_{2}$ is LAS when $\lambda_{4}=\rho z_{2}-\theta u_{2}-\delta_{1}=\rho \beta \sigma_{2} \delta_{3}-$ $\delta_{1} \delta_{3}\left(\omega_{2}^{*}-\omega_{2}\right)-\theta \beta \gamma_{2} \sigma_{2} / \delta_{3}\left(\omega_{2}^{*}-\omega_{2}\right)<0$, which is equivalent to $\theta>\theta_{2}$.

At $P^{*}$, characteristic (13) becomes

$$
\lambda^{4}+A_{1} \lambda^{3}+A_{2} \lambda^{2}+A_{3} \lambda+A_{4}=0
$$

where

$$
\begin{aligned}
A_{1}= & \alpha \beta x^{*}+\delta_{3}+\delta_{2}-\omega_{2} x^{*}>0, \\
A_{2}= & \theta \gamma_{1} y^{*}+\alpha \beta\left(\delta_{2}-\omega_{2} x^{*}\right) x^{*}+\left(\alpha \beta x^{*}+\delta_{2}-\omega_{2} x^{*}\right) \delta_{3}>0, \\
A_{3}= & \theta \gamma_{1}\left(\alpha \beta x^{*}+\delta_{2}-\omega_{2} x^{*}\right) y^{*}+\alpha \beta \delta_{3}\left(\delta_{2}-\omega_{2} x^{*}\right) x^{*} \\
& +\rho \omega_{2} x^{*} y^{*} z^{*}>0, \\
A_{4}= & \theta \alpha \beta \gamma_{1}\left(\delta_{2}-\omega_{2} x^{*}\right) x^{*} y^{*}+\left(\rho \delta_{3}-\theta \gamma_{2}\right) \omega_{2} x^{*} y^{*} z^{*} .
\end{aligned}
$$

Note that $\delta_{2}>\omega_{2} x^{*}$ and $\theta<\min \left\{\theta_{2}, \theta_{3}\right\}$ are necessary for the existence of $P^{*}$, then we have $A_{4}>0$. By the Routh-Hurwitz criterion, the roots of (17) have only negative real parts if and only if

$$
\begin{aligned}
A_{1}>0, A_{2} & >0, A_{3}>0, A_{4}>0, A_{1} A_{2} \\
& -A_{3}>0, A_{1}\left(A_{2} A_{3}-A_{1} A_{4}\right)-A_{3}^{2}>0 .
\end{aligned}
$$

Hence, we obtain the sufficient conditions for stability of $P^{*}:$
(H1): $\delta_{2}>\omega_{2} x^{*}, \theta<\min \left\{\theta_{2}, \theta_{3}\right\}$,

(H2): $A_{1} A_{2}-A_{3}=\theta \gamma_{1}\left(\alpha \beta x^{*}+\delta_{2}-\omega_{2} x^{*}\right) y^{*}$

$$
+\alpha \beta \delta_{3}\left(\delta_{2}-\omega_{2} x^{*}\right) x^{*}
$$$$
-\rho x^{*} y^{*} z^{*}>0,
$$

(H3): $A_{1}\left(A_{2} A_{3}-A_{1} A_{4}\right)-A_{3}^{2}=B_{1} \theta+B_{2}>0$,

where

$$
\begin{aligned}
B_{1}= & \left(\left(\alpha \beta x^{*}+\delta_{2}-\omega_{2} x^{*}\right)^{2} \delta_{3}+\alpha \beta x^{*}+\delta_{2}-\omega_{2} x^{*}\right) \delta_{3}^{2} \\
& -\rho \omega_{2} x^{*} y^{*} z^{*}\left(\alpha \beta x^{*}+\delta_{2}-\omega_{2} x^{*}\right) \gamma_{1} y^{*} \\
- & \left(\alpha \beta x^{*}+\delta_{2}-\omega_{2} x^{*}+\delta_{3}\right)^{2}\left(\alpha \beta \gamma_{1}\left(\delta_{2}-\omega_{2} x^{*}\right) x^{*} y^{*}\right. \\
& \left.-\omega_{2} \gamma_{2} x^{*} y^{*} z^{*}\right), \\
B_{2}= & {\left[\left(\left(\alpha \beta x^{*}+\delta_{2}-\omega_{2} x^{*}\right)^{2} \delta_{3}+\alpha \beta x^{*}+\delta_{2}-\omega_{2} x^{*}\right) \delta_{3}^{2}\right.} \\
& \left.-\rho \omega_{2} x^{*} y^{*} z^{*}\left(\alpha \beta \delta_{3}\left(\delta_{2}-\omega_{2} x^{*}\right) x^{*}+\rho \omega_{2} x^{*} y^{*} z^{*}\right)\right] \\
- & \left(\alpha \beta x^{*}+\delta_{2}-\omega_{2} x^{*}+\delta_{3}\right)^{2} \rho \omega_{2} \delta_{3} x^{*} y^{*} z^{*} .
\end{aligned}
$$

Theorem 4. System (3) has a unique coexistence equilibrium $P^{*}$, when $\omega_{2} \leq \omega_{2}^{*}, \theta_{0}<\theta<\theta_{2}$ or $\omega_{2}>\omega_{2}^{*}, \theta_{0}<\theta<\theta_{3}$ hold, and $P^{*}$ is LAS, when conditions (20) are satisfied.

We summarize the above results in Table 1 , where $\omega_{2}^{*}=\beta \delta_{2}, \theta_{0}=\delta_{3}\left(\rho \sigma_{2}-\delta_{1} \delta_{2}\right) / \alpha \gamma_{1} \delta_{2}+\gamma_{2} \sigma_{2}$,

$\theta_{1}=\delta_{3}\left(\rho \sigma_{2}-\delta_{1} \delta_{2}\right) / \gamma_{2} \sigma_{2}$,

$\theta_{2}=\beta \delta_{3}\left(\rho \sigma_{2}-\delta_{1} \delta_{2}\right)+\delta_{1} \delta_{3} \omega_{2} / \beta \gamma_{2} \sigma_{2}$, and $\theta_{3}=\rho \delta_{3} / \gamma_{2}$.

2.4. Numerical Simulations. In this section, we choose some suitable parameters in (3) to simulate numerically the theoretical conclusions obtained in the previous sections by using the Matlab software package MATCONT [45]. We select the following parameters' set [4]:

$$
\begin{aligned}
& \alpha=1.636, \beta=0.002, \delta_{1}=0.3743, \sigma_{2}=0.38, \\
& \quad \delta_{2}=0.055, \rho=0.48, \gamma_{1}=0.15, \gamma_{2}=0.2, \delta_{3}=0.25 .
\end{aligned}
$$

Note that $\omega_{2}^{*}=\beta \delta_{2}=0.00011$. By calculations, we have 


$$
\begin{aligned}
& \theta_{0}=\frac{\delta_{3}\left(\rho \sigma_{2}-\delta_{1} \delta_{2}\right)}{\alpha \gamma_{1} \delta_{2}+\gamma_{2} \sigma_{2}}=0.452, \\
& \theta_{1}=\frac{\delta_{3}\left(\rho \sigma_{2}-\delta_{1} \delta_{2}\right)}{\gamma_{2} \sigma_{2}}=0.5323 \\
& \theta_{3}=\frac{\rho \delta_{3}}{\gamma_{2}}=0.6 \\
& \theta_{2}=\frac{\beta \delta_{3}\left(\rho \sigma_{2}-\delta_{1} \delta_{3}\right)+\delta_{1} \delta_{3} \omega_{2}}{\beta \gamma_{2} \sigma_{2}}=615.625 \omega_{2}+0.5323
\end{aligned}
$$

And we find the stability region of $P^{*}=\left(x^{*}, y^{*}, z^{*}, u^{*}\right)$ (see Figure 2). $P^{*}$ is LAS in regions I and II. $P^{*}$ is unstable in region III.

Let us denote the point in $\theta-\omega_{2}$ plane as $Q_{i}=\left(\theta, \omega_{2}\right)$.

Case (a): we choose a point $Q_{1}=(0.48,0.0001)$ in the region I; then, system (3) has one interior equilibrium:

$$
P_{1}^{*}=(77.6449,1.38195,8.0448,7.265)
$$

The eigenvalues of Jacobian matrix of (12) are

$$
\begin{aligned}
& -0.4623,-0.049151,-0.01991-0.378 i \text { and } \\
& -0.01991+0.378 i,
\end{aligned}
$$

so $P_{1}^{*}$ is stable, as shown in Figure 3(a).

Case (b): we choose a point $Q_{2}=(0.455,0.0004)$ in the region II; then, system (3) has one interior equilibrium:

$$
P_{2}^{*}=(2.84485,1.62669,7.05506,6.62006) \text {. }
$$

The eigenvalues of Jacobian matrix of (12) are

$$
\begin{aligned}
& -0.0931+0.301 i,-0.0931-0.301 i,-0.0633 \\
& +0.0188 i \text { and }-0.0633-0.0188 i,
\end{aligned}
$$

so $P_{2}^{*}$ is stable, as shown in Figure 3(b).

Case (c): we choose a point $Q_{3}=(0.48,0.000111)$ in the region II, then system (3) has one interior equilibrium:

$$
P_{3}^{*}=(72.569,1.39855,8.094,7.314)
$$

The eigenvalues of Jacobian matrix of (12) are

$-0.458,-0.0491,-0.0132+0.383 i$ and $-0.0132-0.383 i$,

so $P_{3}^{*}$ is stable, as shown in Figure 3(c).

Case $(d)$ : we choose a point $Q_{4}=(0.48,0.0004)$ in the region III; then, system (3) has one interior equilibrium:

$$
P_{4}^{*}=(26.365,1.54973,8.54816,7.76837) .
$$

The eigenvalues of Jacobian matrix of (12) are $-0.4328,-0.0497,0.0509+0.416 i$ and $0.0509-0.416 i$,

so $P_{4}^{*}$ is unstable, as shown in Figure $3(\mathrm{~d})$.

Below, we perform numerically bifurcation analysis of $x$ against $\theta$ for different values of $\omega_{2}$.

Case (1): we choose $\omega_{2}=0.0001<\omega_{2}^{*}=0.00011$ (see Figure 4(a)).

We obtain the following result.

Proposition 2. When $0<\theta<\theta_{0}, P_{0}$ exists and is unstable, $P_{1}$ exists and is LAS, $P_{2}$ exists and is unstable, and $P^{*}$ does not exist. When $\theta_{0}<\theta<\theta_{1}, P_{0}$ exists and is unstable, $P_{1}$ exists and is unstable, $P_{2}$ exists and is unstable, and $P^{*}$ exists and is LAS. When $\theta_{1}<\theta<\theta_{2}, P_{0}$ exists and is unstable, $P_{1}$ does not exist, $P_{2}$ exists and is unstable, and $P^{*}$ exists and is LAS. When $\theta>\theta_{2}, P_{0}$ exists and is unstable, $P_{1}$ does not exist, $P_{2}$ exists and is LAS, and $P^{*}$ does not exist.

Case (2): we choose $\omega_{2}=0.0004>\omega_{2}^{*}=0.00011$ (see Figure 4(b)).

We obtain the following result.

Proposition 3. When $0<\theta<\theta_{0}, P_{0}$ exists and is unstable, $P_{1}$ exists and is LAS, and $P^{*}$ does not exist. When $\theta_{0}<\theta<\theta_{4}$, where $\theta_{4}=0.4657$ is a Hopf bifurcation, $P_{0}$ exists and is unstable, $P_{1}$ exists and is LAS, and $P^{*}$ exists and is LAS. When $\theta_{4}<\theta<\theta_{1}, P_{0}$ exists and is unstable, $P_{1}$ exists and is unstable, and $P^{*}$ exists and is unstable. When $\theta_{1}<\theta<\theta_{3}, P_{0}$ exists and is unstable, $P_{1}$ does not exist, and $P^{*}$ exists and is unstable. When $\theta>\theta_{3}, P_{0}$ exists and is unstable, $P_{1}$ does not exist, and $P^{*}$ does not exist.

Consider the case where HTCs stimulation rate $\left(\omega_{2}\right)$ is low by the presence of identified tumor antigens $\left(\omega_{2}<\omega_{2}^{*}\right)$. When the inhibition rate of Tregs to ECs $\theta$ is lower than $\theta_{0}$, the solution of system (3) approaches $P_{1}$ implying that ECs can still effectively remove TCs. When $\theta_{0}<\theta<\theta_{2}$, the solution of system (3) approaches $P^{*}$ showing the coexistence of TCs and immune cells, which means that the patient can survive with tumors. When $\theta>\theta_{2}$, TCs escape the control of the immune system and develop into malignant tumors.

Next consider the case where HTCs stimulation rate $\left(\omega_{2}\right)$ is high by the presence of identified tumor antigens $\left(\omega_{2}>\omega_{2}^{*}\right)$. When $\theta<\theta_{0}$, the solution of system (3) approaches $P_{1}$ implying that TCs can be effectively removed by ECs. When $\theta_{0}<\theta<\theta_{4}$, the solution of system (3) approaches $P^{*}$ implying that TCs can still be controlled by the immune system. When $\theta>\theta_{4}$, the system has a Hopf bifurcation point and induces a limit cycle (see Figure 3(e)). In the biological sense, it can be understood that the number of TCs presents a periodic change. 


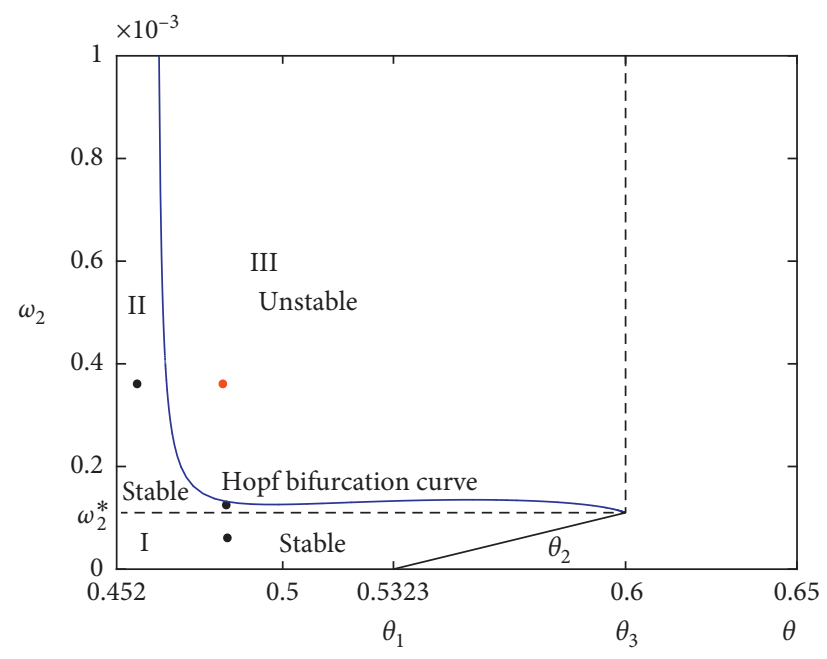

Figure 2: Stability region of $P^{*}$ and Hopf bifurcation curve in $\theta-\omega_{2}$ parameter plane.

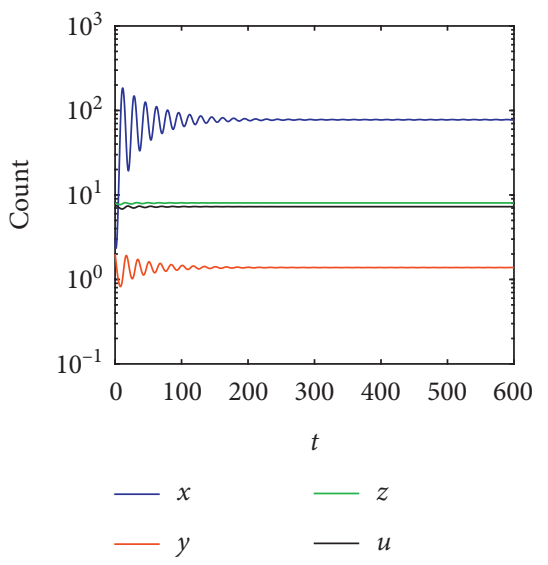

(a)
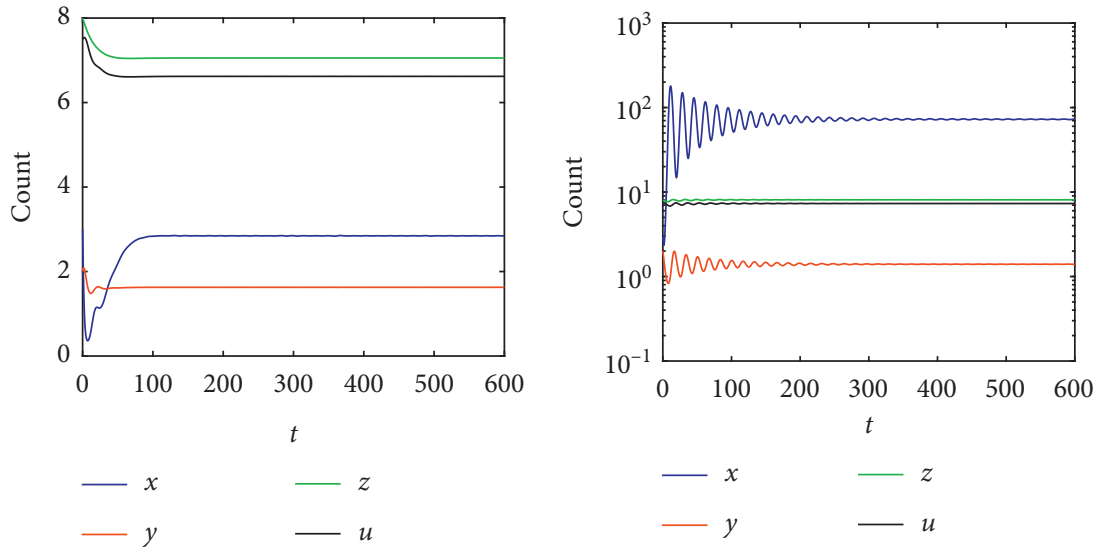

(b)

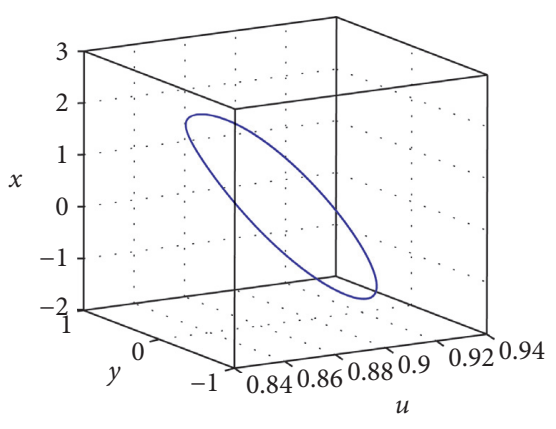

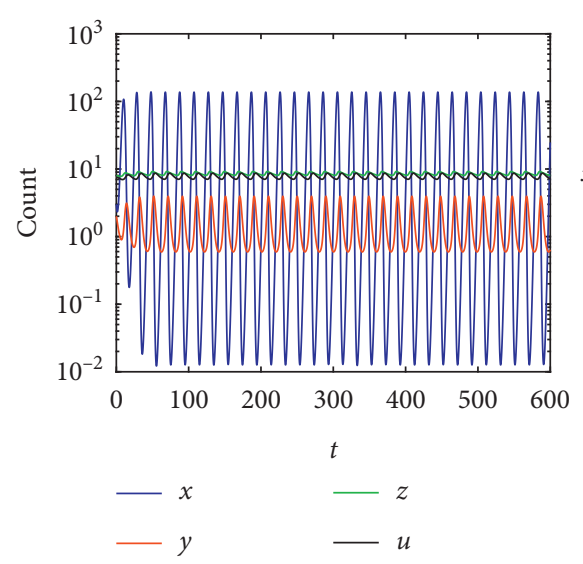

(d) (e)

FIgURE 3: The other parameter values are given in (22). (a) $P_{1}^{*}(77.644,1.381,8.044,7.265)$ is LAS, $\theta=0.48$ and $\omega_{2}=0.0001$. (b) $P_{2}^{*}(2.844,1.626,7.055,6.62)$ is LAS, $\theta=0.455$ and $\omega_{2}=0.0004$. (c) $P_{3}^{*}(72.569,1.398,8.094,7.314)$ is LAS, $\theta=0.48$ and $\omega_{2}=0.000111$. (d) $P_{4}^{*}(26.365,1.549,8.548,7.768)$ is unstable, $\theta=0.48$ and $\omega_{2}=0.0004$. (e) The 3D phase portrait depicts tumor cell, ECs, and Tregs, $\theta=0.48$ and $\omega_{2}=0.0004$. 


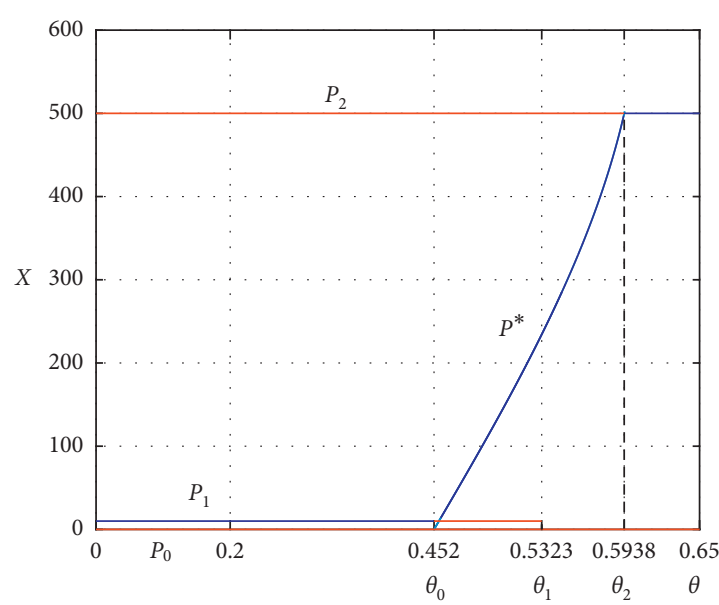

(a)

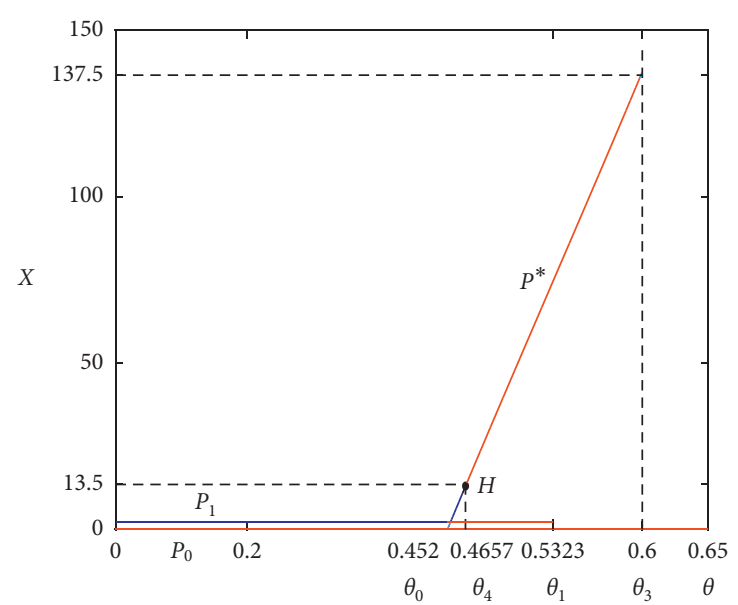

(b)

FIgURE 4: The bifurcation diagrams of $x$ with respect to $\theta$ for different $\omega_{2}=0.0001$ and 0.0004 , respectively. The stable state is represented by the blue curve, and the unstable one corresponds to the red curve. The equilibria are $P_{0}\left(0,0, z_{0}, u_{0}\right), P_{1}\left(0, y_{1}, z_{1}, u_{1}\right), P_{2}\left(500,0, z_{2}, u_{2}\right)$, and $P^{*}\left(x^{*}, y^{*}, z^{*}, u^{*}\right)$ of system (3) (in order to distinguish $P_{0}$ from $P_{1}$, we move $P_{1}$ up a little bit). (a) $\omega_{2}=0.0001$. (b) $\omega_{2}=0.0004$.

\section{Treatment Model}

In order to investigate well the effect of Tregs in tumor immune response under the treatment, we follow the way in [11] to introduce the constant treatment term $s_{1}$ into the second equation of (1). Since CTLA-4 and PD-1 can inhibit the development of Tregs and prevent the transformation of HTCs into Tregs [12], the effect of CTLA4 or PD-1 on Tregs can be considered. Then, we shall establish a five-dimensional ODEs model described as below:

$$
\left\{\begin{array}{l}
\frac{d T(t)}{d t}=a T(t)(1-b T(t))-n E(t) T(t), \\
\frac{d E(t)}{d t}=s_{1}+p E(t) H(t)-q R(t) E(t)-d_{1} E(t), \\
\frac{d H(t)}{d t}=s_{2}+k_{2} T(t) H(t)-d_{2} H(t), \\
\frac{d R(t)}{d t}=r_{1} E(t)+r_{2} H(t)-d_{3} R-m R(t) W(t), \\
\frac{d W(t)}{d t}=s_{3}-d_{4} W(t),
\end{array}\right.
$$

where $W(t)$ represents the concentration of monoclonal antibody in human body at time $t, s_{1}$ represents the treatment term of introducing LAK and TIL into the region of tumor localization, $m$ represents the inhibition rate of monoclonal antibody on Tregs, $s_{3}$ represents the amount of monoclonal antibody entering human body at time $t$, and $d_{4}$ represents the attenuation coefficient of monoclonal antibody.

We scale those new parameters in model (32) as follows:

$$
W(t)=W_{0} v(\tau), \sigma_{1}=\frac{s_{1}}{n E_{0} T_{0}}, \xi=\frac{m W_{0}}{n T_{0}}, \sigma_{3}=\frac{s_{3}}{n W_{0} T_{0}}, \delta_{4}=\frac{d_{4}}{n T_{0}},
$$

and we choose the scaling $W_{0}=10^{6}$. By replacing $\tau$ by $t$, we obtain the following scaled model with treatment:

$$
\left\{\begin{array}{l}
\frac{d x(t)}{d t}=\alpha x(t)(1-\beta x(t))-x(t) y(t), \\
\frac{d y(t)}{d t}=\sigma_{1}+\rho y(t) z(t)-\theta y(t) u(t)-\delta_{1} y(t), \\
\frac{d z(t)}{d t}=\sigma_{2}+\omega_{2} x(t) z(t)-\delta_{2} z(t), \\
\frac{d u(t)}{d t}=\gamma_{1} y(t)+\gamma_{2} z(t)-\delta_{3} u(t)-\xi u(t) v(t), \\
\frac{d v(t)}{d t}=\sigma_{3}-\delta_{4} v(t),
\end{array}\right.
$$

with initial conditions

$$
\begin{aligned}
x(0) & =x_{0} \geq 0, y(0)=y_{0} \geq 0, z(0)=z_{0} \geq 0, u(0) \\
& =u_{0} \geq 0, v(0)=v_{0} \geq 0 .
\end{aligned}
$$

Here, $v$ denotes the dimensionless concentration of monoclonal antibody.

3.1. Model Analysis. By a similar proof of Proposition 1, we can obtain the well posedness of model (34) with initial conditions (35) as follows.

Proposition 4. The solutions $(x(t), y(t), z(t), u(t))$ of model (34) with initial conditions (35) are existent, unique, and nonnegative on the interval $[0,+\infty)$. 
Next, we discuss the effect of three types of immunotherapy on tumors such as

Case I: ACI model $\left(\sigma_{1}>0, \sigma_{3}=0\right)$

Case II: MAI model $\left(\sigma_{1}=0, \sigma_{3}>0\right)$

Case III: combined immunotherapy model $\left(\sigma_{1}>0, \sigma_{3}>0\right)$

We set $\quad d x / d t=0, d y / d t \quad=0, d z / d t=0, d u / d t=$ 0 , and $d v / d t=0$ in (34), and we have

$$
\left\{\begin{array}{l}
\alpha x(1-\beta x)-x y=0, \\
\rho y z-\theta y u-\delta_{1} y+\sigma_{1}=0, \\
\sigma_{2}+\omega_{2} x z-\delta_{2} z=0 \\
\gamma_{1} y+\gamma_{2} z-\delta_{3} u-\xi u v=0 \\
\sigma_{3}-\delta_{4} v=0
\end{array}\right.
$$

3.1.1. ACI Model. When $\sigma_{1}>0$ and $\sigma_{3}=0$, we put $v=0$ and (36) becomes

$$
\left\{\begin{array}{l}
\alpha x(1-\beta x)-x y=0, \\
\rho y z-\theta y u-\delta_{1} y+\sigma_{1}=0, \\
\sigma_{2}+\omega_{2} x z-\delta_{2} z=0 \\
\gamma_{1} y+\gamma_{2} z-\delta_{3} u=0 .
\end{array}\right.
$$

Putting $x=0$ yields the tumor-free equilibrium, namely,

$$
E_{0}=\left(x_{0}, y_{0}, z_{0}, u_{0}\right)=\left(0, y_{0}, \frac{\sigma_{2}}{\delta_{2}}, \frac{\gamma_{1} \delta_{2} y_{0}+\gamma_{2} \sigma_{2}}{\delta_{2} \delta_{3}}\right),
$$

where $y_{0}=-B+\sqrt{B^{2}-4 A C} / 2 A, A=\theta \gamma_{1} \delta_{2}>0, B=\delta_{1} \delta_{2} \delta_{3}$ $+\theta \gamma_{2} \sigma_{2}-\rho \sigma_{2} \delta_{3}$, and $C=-\sigma_{1} \delta_{2} \delta_{3}<0 . E_{0}$ always exists.

Putting $x \neq 0$ and eliminating $y, z$, and $u$ in (37), we have

$$
\begin{aligned}
F(x)= & C_{3} x^{3}+C_{2} x^{2}+C_{1} x+C_{0} \\
C_{3}= & \theta \alpha^{2} \beta^{2} \gamma_{1} \omega_{2}>0, \\
C_{2}= & -\theta \alpha^{2} \beta \gamma_{1} \omega_{2}-\alpha \beta\left[\theta \alpha \gamma_{1}\left(\beta \delta_{2}+\omega_{2}\right)+\delta_{1} \delta_{3} \omega_{2}\right]<0, \\
C_{1}= & \theta \alpha\left(2 \alpha \beta \gamma_{1} \delta_{2}+\alpha \gamma_{1} \omega_{2}+\beta \gamma_{2} \sigma_{2}\right) \\
& +\alpha \delta_{3}\left[\delta_{1} \omega_{2}-\beta\left(\rho \sigma_{2}-\delta_{1} \delta_{2}\right)\right]-\sigma_{1} \omega_{2} \delta_{3}, \\
C_{0}= & \sigma_{1} \delta_{2} \delta_{3}+\alpha \delta_{3}\left(\rho \sigma_{2}-\delta_{1} \delta_{2}\right)-\theta \alpha\left(\alpha \gamma_{1} \delta_{2}+\gamma_{2} \sigma_{2}\right) .
\end{aligned}
$$

Case (a): if $F(0) F(\mu)=C_{0} F(\mu)<0 \quad$ (where $\mu=\min \left\{(1 / \beta),\left(\delta_{2} / \omega_{2}\right)\right\}$, due to $F(+\infty)>0$ and $F(-\infty)<0$, then (39) has one positive root $x^{*}$ in the interval $[0, \mu]$. Therefore, system (34) has a unique coexistence equilibrium:

$$
\begin{aligned}
E^{*}= & \left(x^{*}, y^{*}, z^{*}, u^{*}\right)=\left(x^{*}, \alpha\left(1-\beta x^{*}\right), \frac{\sigma_{2}}{\delta_{2}-\omega_{2} x^{*}},\right. \\
& \left.\frac{\alpha \gamma_{1}\left(1-\beta x^{*}\right)\left(\delta_{2}-\omega_{2} x^{*}\right)+\gamma_{2} \sigma_{2}}{\delta_{3}\left(\delta_{2}-\omega_{2} x^{*}\right)}\right) .
\end{aligned}
$$

We show the existence conditions of $E^{*}$ in the following:

Case (1): $C_{0}>0, F(\mu)<0$.

Since $C_{0}>0$, we have $\theta<\sigma_{1} \delta_{2} \delta_{3}+\alpha \delta_{3}\left(\rho \sigma_{2}-\delta_{1} \delta_{2}\right) /$ $\alpha\left(\alpha \gamma_{1} \delta_{2}+\gamma_{2} \sigma_{2}\right) \triangleq \theta_{5}$. Since $F(\mu)<0$, we have the following two cases:

Case where $1 / \beta<\delta_{2} / \omega_{2}$, i.e., $\omega_{2}<\beta \delta_{2}$. Since $\mu=1 / \beta$, we have $F(\mu)=\delta_{3} \sigma_{1}\left(\beta \delta_{2}-\omega_{2}\right) / \beta>0$. That is a contradiction.

Case where $1 / \beta>\delta_{2} / \omega_{2}$, i.e., $\omega_{2}>\beta \delta_{2}$. Since $\mu=\delta_{2} / \omega_{2}$, we have $F(\mu)=\alpha \sigma_{2}\left(\beta \delta_{2}-\omega_{2}\right)\left(\gamma_{2} \theta-\delta_{3} \rho\right) / \omega_{2}<0$ implying $\theta>\rho \delta_{3} / \gamma_{2}=\theta_{3}$. If $\theta_{5} \geq \theta_{3}$, i.e., $\sigma_{1} \delta_{2} \delta_{3}+$ $\alpha \delta_{3}\left(\rho \sigma_{2}-\delta_{1} \delta_{2}\right) / \alpha\left(\alpha \gamma_{1} \delta_{2}+\gamma_{2} \sigma_{2}\right) \geq \rho \delta_{3} / \gamma_{2}$, we have $\sigma_{1} \geq \alpha\left(\alpha \rho \gamma_{1}+\delta_{1} \gamma_{2}\right) / \gamma_{2}$, there exists $E^{*}$.

Case (2): $C_{0}<0, F(\mu)>0$.

Since $C_{0}<0$, we have $\theta>\theta_{5}$. Since $F(\mu)>0$, we have the following two cases:

Case where $1 / \beta<\delta_{2} / \omega_{2}$, i.e., $\omega_{2}<\beta \delta_{2}$. Since $\mu=1 / \beta$, we have $F(\mu)=\delta_{3} \sigma_{1}\left(\beta \delta_{2}-\omega_{2}\right) / \beta>0$, there exists $E^{*}$. Case where $1 / \beta>\delta_{2} / \omega_{2}$, i.e., $\omega_{2}>\beta \delta_{2}$. Since $\mu=\delta_{2} / \omega_{2}$, we have $F(\mu)=\alpha \sigma_{2}\left(\beta \delta_{2}-\omega_{2}\right)\left(\gamma_{2} \theta-\delta_{3} \rho\right) / \omega_{2}>0$ implying $\theta<\theta_{3}$. If $\theta_{3} \geq \theta_{5}$, i.e., $\rho \delta_{3} / \gamma_{2} \geq \sigma_{1} \delta_{2} \delta_{3}+$ $\alpha \delta_{3}\left(\rho \sigma_{2}-\delta_{1} \delta_{2}\right) / \alpha\left(\alpha \gamma_{1} \delta_{2}+\gamma_{2} \sigma_{2}\right)$, we have $\sigma_{1} \leq \alpha$ $\left(\alpha \rho \gamma_{1}+\delta_{1} \gamma_{2}\right) / \gamma_{2}$, there exists $E^{*}$.

Case (b): If $F(0) F(\mu)=C_{0} F(\mu)>0$, then (39) can have two positive roots $x_{1}^{*}$ and $x_{2}^{*}$ in the interval $[0, \mu]$, where $x_{1}^{*}$ and $x_{2}^{*}$ are the roots of (39). Therefore, system (34) can have two coexistence equilibria:

$$
\begin{aligned}
E_{1}^{*}= & \left(x_{1}^{*}, y_{1}^{*}, z_{1}^{*}, u_{1}^{*}\right)=\left(x_{1}^{*}, \alpha\left(1-\beta x_{1}^{*}\right), \frac{\sigma_{2}}{\delta_{2}-\omega_{2} x_{1}^{*}},\right. \\
& \left.\frac{\alpha \gamma_{1}\left(1-\beta x_{1}^{*}\right)\left(\delta_{2}-\omega_{2} x_{1}^{*}\right)+\gamma_{2} \sigma_{2}}{\delta_{3}\left(\delta_{2}-\omega_{2} x_{1}^{*}\right)}\right), \\
E_{2}^{*}= & \left(x_{2}^{*}, y_{2}^{*}, z_{2}^{*}, u_{2}^{*}\right)=\left(x_{2}^{*}, \alpha\left(1-\beta x_{2}^{*}\right), \frac{\sigma_{2}}{\delta_{2}-\omega_{2} x_{2}^{*}},\right. \\
& \left.\frac{\alpha \gamma_{1}\left(1-\beta x_{2}^{*}\right)\left(\delta_{2}-\omega_{2} x_{2}^{*}\right)+\gamma_{2} \sigma_{2}}{\delta_{3}\left(\delta_{2}-\omega_{2} x_{2}^{*}\right)}\right) .
\end{aligned}
$$

In the following, we show the existence condition of $E_{1}^{*}$ and $E_{2}^{*}$. 
Differentiating (39), we have

$$
F^{\prime}(x)=3 C_{3} x^{2}+2 C_{2} x+C_{1}=0,
$$

where $C_{3}>0$ and $C_{2}<0$. If $C_{1}>0$ and $\Delta \triangleq C_{2}^{2}-3 C_{1} C_{3}>0$, then (43) has two positive roots $x_{1}^{+}=-C_{2}-\sqrt{\Delta} / 3 C_{3}$ and $x_{2}^{+}=-C_{2}+\sqrt{\Delta} / 3 C_{3}$. And if $C_{1}<0$, then (43) has one positive root $x_{2}^{+}=-C_{2}+\sqrt{\Delta} / 3 C_{3}$.

Case (1): $C_{0}>0, F(\mu)>0$.

Since $C_{0}>0$, we have $\theta<\theta_{5}$. Since $F(\mu)>0$, we have the following two cases:

Case where $1 / \beta<\delta_{2} / \omega_{2}$, i.e., $\omega_{2}<\beta \delta_{2}$. Since $\mu=1 / \beta$, we have $F(\mu)=\delta_{3} \sigma_{1}\left(\beta \delta_{2}-\omega_{2}\right) / \beta>0$. If $F\left(x_{2}^{+}\right)<0$, then there exist $E_{1}^{*}$ and $E_{2}^{*}$.

Case where $1 / \beta>\delta_{2} / \omega_{2}$, i.e., $\omega_{2}>\beta \delta_{2}$. Since $\mu=\delta_{2} / \omega_{2}$, we have $F(\mu)=\alpha \sigma_{2}\left(\beta \delta_{2}-\omega_{2}\right)\left(\gamma_{2} \theta-\delta_{3} \rho\right) / \omega_{2}>0$ implying $\theta<\theta_{3}$. Then, we have $\theta<\min \left\{\theta_{3}, \theta_{5}\right\}$. If $F\left(x_{2}^{+}\right)<0$, then there exist $E_{1}^{*}$ and $E_{2}^{*}$.

Case (2): $C_{0}<0, F(\mu)<0$.

Since $C_{0}<0$, we have $\theta>\theta_{5}$. Since $F(\mu)>0$, we have the following two cases:

Case where $1 / \beta<\delta_{2} / \omega_{2}$, i.e., $\omega_{2}<\beta \delta_{2}$. Since $\mu=1 / \beta$, we have $F(\mu)=\delta_{3} \sigma_{1}\left(\beta \delta_{2}-\omega_{2}\right) / \beta>0$. That is a contradiction.

Case where $1 / \beta>\delta_{2} / \omega_{2}$, i.e., $\omega_{2}>\beta \delta_{2}$. Since $\mu=\delta_{2} / \omega_{2}$, we have $F(\mu)=\alpha \sigma_{2}\left(\beta \delta_{2}-\omega_{2}\right)\left(\gamma_{2} \theta-\delta_{3} \rho\right) / \omega_{2}<0 \quad$ implying $\theta>\theta_{3}$. Then, we have $\theta>\max \left\{\theta_{3}, \theta_{5}\right\}$. If $F\left(x_{1}^{+}\right)>0$, then there exist $E_{1}^{*}$ and $E_{2}^{*}$.

Next, we show the stability of the above two equilibria $E_{0}$ and $E^{*}$ of system (34) with $v=0$. We linearize the system and obtain the characteristic equation whose expression is the same as (13).

Theorem 5. System (34) with $v=0$ always has one tumorfree equilibrium $E_{0}$, which is LAS if the inequality $\theta<\theta_{5}$ holds.

Proof. At $E_{0}=\left(x_{0}, y_{0}, z_{0}, u_{0}\right)=\left(0, y_{0}, \sigma_{2} / \delta_{2}, \gamma_{1} \delta_{2} y_{0}+\right.$ $\left.\gamma_{2} \sigma_{2} / \delta_{2} \delta_{3}\right)$, the characteristic equation becomes

$$
\left(\lambda-\alpha+y_{0}\right)\left(\lambda+\delta_{2}\right)\left(\lambda^{2}+\left(\frac{\sigma_{1}}{y_{0}}+\delta_{3}\right) \lambda+\theta \gamma_{1} y_{0}+\frac{\sigma_{1} \delta_{3}}{y_{0}}\right)=0
$$

and one root of characteristic equation is $-\delta_{2}<0$. It is easily noted that as $\sigma_{1} / y_{0}+\delta_{3}>0, \theta \gamma_{1} y_{1}+\sigma_{1} \delta_{3} / y_{0}>0$, so the solutions of $\lambda^{2}+\left(\left(\sigma_{1} / y_{0}\right)+\delta_{3}\right) \lambda+\theta \gamma_{1} y_{1}+\left(\sigma_{1} \delta_{3} / y_{0}\right)=0$ have always negative real parts. Therefore, $E_{0}$ is LAS if and only if $\alpha-y_{0}=2 \alpha A+B-\sqrt{B^{2}-4 A C} / 2 A<0$, that is, if and only if $\theta<\theta_{5}$.

At $E^{*}=\left(x^{*}, y^{*}, z^{*}, u^{*}\right)$, characteristic (13) becomes

$$
\lambda^{4}+B_{1} \lambda^{3}+B_{2} \lambda^{2}+B_{3} \lambda+B_{4}=0
$$

$$
\begin{aligned}
B_{1}= & \alpha \beta x^{*}+\delta_{3}+\frac{\sigma_{1}}{y^{*}}+\frac{\sigma_{2}}{z^{*}}>0, \\
B_{2}= & \left(\alpha \beta x^{*}+\frac{\sigma_{2}}{z^{*}}\right)\left(\frac{\sigma_{1}}{y^{*}}+\delta_{3}\right)+\alpha \beta x^{*} \frac{\sigma_{2}}{z^{*}}+\delta_{3} \frac{\sigma_{1}}{y^{*}}+\theta \gamma_{1} y^{*}>0, \\
B_{3}= & \left(\theta \gamma_{1} y^{*}+\delta_{3} \frac{\sigma_{1}}{y^{*}}\right)\left(\alpha \beta x^{*}+\frac{\sigma_{2}}{z^{*}}\right)+\alpha \beta x^{*} \frac{\sigma_{2}}{z^{*}}\left(\delta_{3}+\frac{\sigma_{1}}{y^{*}}\right) \\
& +\rho \omega_{2} x^{*} y^{*} z^{*}>0, \\
B_{4}= & \alpha \beta x^{*} \frac{\sigma_{2}}{z^{*}}\left(\theta \gamma_{1} y^{*}+\delta_{3} \frac{\sigma_{1}}{y^{*}}\right)+\left(\rho \delta_{3}-\theta \gamma_{2}\right) \omega_{2} x^{*} y^{*} z^{*} .
\end{aligned}
$$

By the Routh-Hurwitz criterion, the roots of (45) have negative real parts if and only if $B_{1}>0, B_{2}>0, B_{3}>0$, $B_{4}>0, B_{1} B_{2}-B_{3}>0$, and $B_{1}\left(B_{2} B_{3}-B_{1} B_{4}\right)-B_{3}^{2}>0$.

Hence, we obtain the sufficient condition for the stability of $P^{*}$ :

$$
\begin{aligned}
(H 4): B_{4}= & \alpha \beta x^{*} \frac{\sigma_{2}}{z^{*}}\left(\theta \gamma_{1} y^{*}+\delta_{3} \frac{\sigma_{1}}{y^{*}}\right) \\
& +\left(\rho \delta_{3}-\theta \gamma_{2}\right) \omega_{2} x^{*} y^{*} z^{*}>0,
\end{aligned}
$$

(H5): $B_{1} B_{2}-B_{3}=K_{1} \theta+K_{2}>0$,

(H6): $B_{1}\left(B_{2} B_{3}-B_{1} B_{4}\right)-B_{3}^{2}=B_{3}\left(K_{1} \theta+K_{2}\right)-B_{1}^{2} B_{4}$

where

$$
\begin{aligned}
K_{1}= & \left(\sigma_{1}+\delta_{3} y^{*}\right) \gamma_{1}, \\
K_{2}= & \left(\delta_{3}+\frac{\sigma_{1}}{y^{*}}+\frac{\sigma_{2}}{z^{*}}\right)\left[\alpha^{2} \beta^{2} x^{* 2}+\left(\alpha \beta x^{*}+\frac{\sigma_{2}}{z^{*}}\right)\left(\frac{\sigma_{1}}{y^{*}}+\delta_{3}\right)\right. \\
& \left.+\alpha \beta x^{*} \frac{\sigma_{2}}{z^{*}}\right]+\delta_{3} \frac{\sigma_{1}}{y^{*}}\left(\frac{\sigma_{1}}{y^{*}}+\delta_{3}\right)-\rho \omega_{2} x^{*} y^{*} z^{*}, \\
K_{3}= & K_{1}\left(\alpha \beta x^{*}+\frac{\sigma_{2}}{z^{*}}\right) \gamma_{1} y^{*}, \\
K_{4}= & \left.\left(K_{2} \gamma_{1} y^{*}+K_{1} \frac{\sigma_{1} \delta_{3}}{y^{*}}\right)\left(\alpha \beta x^{*}+\frac{\sigma_{2}}{z^{*}}\right)\right] \\
& +K_{1}\left[\alpha \beta x^{*} \frac{\sigma_{2}}{z^{*}}\left(\delta_{3}+\frac{\sigma_{1}}{y^{*}}-\left(\alpha \beta x^{*}+\delta_{3}+\frac{\sigma_{1}}{y^{*}}+\frac{\sigma_{2}}{z^{*}}\right)^{2} \gamma_{1} y^{*}\right)\right. \\
& \left.+\left(\rho+\gamma_{2}\right) \omega_{2} x^{*} y^{*} z^{*}\right], \\
K_{5}= & K_{2}\left(\alpha \beta x^{*}+\frac{\sigma_{2}}{z^{*}}\right) \frac{\sigma_{1} \delta_{3}}{y^{*}}-\alpha \beta x^{*} \frac{\sigma_{2}}{z^{*}}\left[K_{2}\left(\delta_{3}+\frac{\sigma_{1}}{y^{*}}\right)\right. \\
& \left.-\left(\alpha \beta x^{*}+\delta_{3}+\frac{\sigma_{1}}{y^{*}}+\frac{\sigma_{2}}{z^{*}}\right)^{2} \frac{\sigma_{1} \delta_{3}}{y^{*}}\right]+\left(K_{2}-\delta_{3}\right) \rho \omega_{2} x^{*} y^{*} z^{*} . \\
& (\alpha)
\end{aligned}
$$

where 
Theorem 6. System (34) with $v=0$ has a coexistence equilibrium $E^{*}$ when $\omega_{2}>\beta \delta_{2}, \sigma_{1} \geq \alpha\left(\alpha \rho \gamma_{1}+\delta_{1} \gamma_{2}\right) / \gamma_{2}$, $\theta_{3}<\theta<\theta_{5}$ or $\omega_{2}>\beta \delta_{2}, \sigma_{1} \leq \alpha\left(\alpha \rho \gamma_{1}+\delta_{1} \gamma_{2}\right) / \gamma_{2}, \theta_{5}<\theta<\theta_{3}$ or $\omega_{2}<\beta \delta_{2}, \theta>\theta_{5}$ hold. Further $E^{*}$ is LAS when condition (47) is satisfied.

3.1.2. MAI Model. When $\sigma_{1}=0, \sigma_{3}>0$, (36) becomes

$$
\left\{\begin{array}{l}
\alpha x(1-\beta x)-x y=0 \\
\rho y z-\theta y u-\delta_{1} y=0 \\
\sigma_{2}+\omega_{2} x z-\delta_{2} z=0 \\
\gamma_{1} y+\gamma_{2} z-\delta_{3} u-\xi u v=0 \\
\sigma_{3}-\delta_{4} v=0
\end{array}\right.
$$

Equation (49) can be simplified as follows:

$$
\left\{\begin{array}{l}
\alpha x(1-\beta x)-x y=0 \\
\rho y z-\theta y u-\delta_{1} y=0 \\
\sigma_{2}+\omega_{2} x z-\delta_{2} z=0 \\
\gamma_{1} y+\gamma_{2} z-\delta_{31} u=0
\end{array}\right.
$$

where $\delta_{31}=\delta_{3} \delta_{4}+\xi \sigma_{3} / \delta_{4}$.

The analysis of (50) is similar to that of (12), and the equilibria of (34) can be obtained as follows:

$$
\begin{aligned}
F_{0}= & \left(0,0, \frac{\sigma_{2}}{\delta_{2}}, \frac{\gamma_{2} \sigma_{2}}{\delta_{2} \delta_{31}}, \frac{\sigma_{3}}{\delta_{4}}\right) \\
= & \left(0, \frac{\delta_{31}\left(\rho \sigma_{2}-\delta_{1} \delta_{2}\right)-\theta \gamma_{2} \sigma_{2}}{\theta \gamma_{1} \delta_{2}}, \frac{\sigma_{2}}{\delta_{2}}, \frac{\rho \sigma_{2}-\delta_{1} \delta_{2}}{\theta \delta_{2}}, \frac{\sigma_{3}}{\delta_{4}}\right), \\
F_{2}= & \left(\frac{1}{\beta}, 0, \frac{\beta \sigma_{2}}{\beta \delta_{2}-\omega_{2}}, \frac{\beta \gamma_{2} \sigma_{2}}{\delta_{31}\left(\beta \delta_{2}-\omega_{2}\right)}, \frac{\sigma_{3}}{\delta_{4}}\right), \\
F^{*}= & \left(x^{*}, \alpha\left(1-\beta x^{*}\right), \frac{\sigma_{2}}{\delta_{2}-\omega_{2} x^{*}},\right. \\
& \left.\frac{\alpha \gamma_{1}\left(1-\beta x^{*}\right)\left(\delta_{2}-\omega_{2} x^{*}\right)+\gamma_{2} \sigma_{2}}{\delta_{31}\left(\delta_{2}-\omega_{2} x^{*}\right)}, \frac{\sigma_{3}}{\delta_{4}}\right) .
\end{aligned}
$$

Here, $x^{*} \in(0, \mu)$ and satisfies the equation $a_{2} x^{2}+a_{1} x+a_{0}=0$, where coefficients are defined as $a_{2}=$ $-\theta \alpha \beta \gamma_{1} \omega_{2}<0, a_{1}=\theta \alpha \gamma_{1}\left(\beta \delta_{2}+\omega_{2}\right)+\delta_{1} \delta_{31} \omega_{2}>0$, and $a_{0}=$ $\delta_{31}\left(\rho \sigma_{2}-\delta_{1} \delta_{2}\right)-\theta\left(\alpha \gamma_{1} \delta_{2}+\gamma_{2} \sigma_{2}\right)$.

The Jacobian matrix of system (34) at any equilibrium $\bar{F}(\bar{x}, \bar{y}, \bar{z}, \bar{u}, \bar{v})$ is as follows:

$$
\mathbf{J}(\bar{F})=\left(\begin{array}{ccccc}
\alpha-2 \alpha \beta \bar{x}-\bar{y} & -\bar{x} & 0 & 0 & 0 \\
0 & \rho \bar{z}-\theta \bar{u}-\delta_{1} & \rho \bar{y} & -\theta \bar{y} & 0 \\
\omega_{2} \bar{z} & 0 & \omega_{2} \bar{x}-\delta_{2} & 0 & 0 \\
0 & \gamma_{1} & \gamma_{2} & -\delta_{3}-\xi \bar{v} & \xi \bar{u} \\
0 & 0 & 0 & 0 & -\delta_{4}
\end{array}\right)=\left(\begin{array}{ccccc}
\alpha-2 \alpha \beta \bar{x}-\bar{y} & -\bar{x} & 0 & 0 & 0 \\
0 & \rho \bar{z}-\theta \bar{u}-\delta_{1} & \rho \bar{y} & -\theta \bar{y} & 0 \\
\omega_{2} \bar{z} & 0 & \omega_{2} \bar{x}-\delta_{2} & 0 & 0 \\
0 & \gamma_{1} & \gamma_{2} & -\delta_{31} & \xi \bar{u} \\
0 & 0 & 0 & 0 & -\delta_{4}
\end{array}\right) .
$$

The corresponding characteristic equation is

$$
|\mathbf{J}(\bar{F})-\lambda \mathbf{I}|=0 .
$$

Substituting $\delta_{31}$ for $\delta_{3}$ in (12), we have

$$
|\mathbf{J}(\bar{F})-\lambda \mathbf{I}|=\left(\lambda+\delta_{4}\right)|\mathbf{J}(\bar{P})-\lambda \mathbf{I}|=0 .
$$

Thus, the stability analysis of $\bar{F}$ is similar to that of $\bar{P}$.

Substituting $\delta_{31}$ for $\delta_{3}$ in (20) to get new conditions for the stability of $F^{*}$ :

$$
\begin{aligned}
\text { (h1): } A_{4}= & \theta \alpha \beta \gamma_{1}\left(\delta_{2}-\omega_{2} x^{*}\right) x^{*} y^{*} \\
& +\left(\rho \delta_{31}-\theta \gamma_{2}\right) \omega_{2} x^{*} y^{*} z^{*}>0, \\
(h 2): A_{1} A_{2}-A_{3}= & \theta \gamma_{1}\left(\alpha \beta x^{*}+\delta_{2}-\omega_{2} x^{*}\right) y^{*} \\
& +\alpha \beta \delta_{31}\left(\delta_{2}-\omega_{2} x^{*}\right) x^{*} \\
& -\rho x^{*} y^{*} z^{*}>0,
\end{aligned}
$$

(h3): $A_{1}\left(A_{2} A_{3}-A_{1} A_{4}\right)-A_{3}^{2}=B_{1} \theta+B_{2}>0$, where

$$
\begin{aligned}
B_{1}= & \left(\left(\alpha \beta x^{*}+\delta_{2}-\omega_{2} x^{*}\right)^{2} \delta_{31}+\left(\alpha \beta x^{*}+\delta_{2}-\omega_{2} x^{*}\right) \delta_{31}^{2}\right. \\
& \left.-\rho \omega_{2} x^{*} y^{*} z^{*}\right)\left(\alpha \beta x^{*}+\delta_{2}-\omega_{2} x^{*}\right) \gamma_{1} y^{*} \\
& -\left(\alpha \beta x^{*}+\delta_{2}-\omega_{2} x^{*}+\delta_{31}\right)^{2}\left(\alpha \beta \gamma_{1}\left(\delta_{2}-\omega_{2} x^{*}\right) x^{*} y^{*}\right. \\
& \left.-\omega_{2} \gamma_{2} x^{*} y^{*} z^{*}\right), \\
B_{2}= & \left(\left(\alpha \beta x^{*}+\delta_{2}-\omega_{2} x^{*}\right)^{2} \delta_{31}+\left(\alpha \beta x^{*}+\delta_{2}-\omega_{2} x^{*}\right) \delta_{31}^{2}\right. \\
& \left.-\rho \omega_{2} x^{*} y^{*} z^{*}\right)\left(\alpha \beta \delta_{31}\left(\delta_{2}-\omega_{2} x^{*}\right) x^{*}+\rho \omega_{2} x^{*} y^{*} z^{*}\right) \\
& -\left(\alpha \beta x^{*}+\delta_{2}-\omega_{2} x^{*}+\delta_{31}\right)^{2} \rho \omega_{2} \delta_{31} x^{*} y^{*} z^{*} .
\end{aligned}
$$

We set 


$$
\begin{aligned}
& \theta_{70}=\frac{\delta_{31}\left(\rho \sigma_{2}-\delta_{1} \delta_{2}\right)}{\alpha \gamma_{1} \delta_{2}+\gamma_{2} \sigma_{2}}, \\
& \theta_{71}=\frac{\delta_{31}\left(\rho \sigma_{2}-\delta_{1} \delta_{2}\right)}{\gamma_{2} \sigma_{2}}, \\
& \theta_{72}=\frac{\beta \delta_{31}\left(\rho \sigma_{2}-\delta_{1} \delta_{2}\right)+\delta_{1} \delta_{31} \omega_{2}}{\beta \gamma_{2} \sigma_{2}}, \\
& \theta_{73}=\frac{\rho \delta_{31}}{\gamma_{2}},
\end{aligned}
$$

and we obtain the following results.

Theorem 7. System (34) always has one tumor-free and ECsfree equilibrium $F_{0}$, which is unstable.

Theorem 8. System (34) has one tumor-free equilibrium $F_{1}$ when $\rho>\delta_{1} \delta_{2} / \sigma_{2}$ and $\theta<\theta_{71}$, which is LAS if the inequality $\theta<\theta_{70}$ holds.

Theorem 9. System (34) has one tumor-dominant equilibrium $F_{2}$ when $\omega_{2}<\omega_{2}^{*}$, which is LAS if the inequality $\theta>\theta_{72}$ holds.

Theorem 10. System (34) has a unique coexistence equilibrium $F^{*}$ when $\omega_{2}<\omega_{2}^{*}, \theta_{0}<\theta<\theta_{2}$ or $\omega_{2}>\omega_{2}^{*}, \theta_{0}<\theta<\theta_{3}$ hold. Further $F^{*}$ is LAS, when condition (55) is satisfied.

3.1.3. Combined Immunotherapy Model. When $\sigma_{1}>0$ and $\sigma_{3}>0,(36)$ can be simplified as follows:

$$
\left\{\begin{array}{l}
\alpha x(1-\beta x)-x y=0 \\
\rho y z-\theta y u-\delta_{1} y+\sigma_{1}=0 \\
\sigma_{2}+\omega_{2} x z-\delta_{2} z=0 \\
\gamma_{1} y+\gamma_{2} z-\delta_{31} u=0
\end{array}\right.
$$

where $\delta_{31}=\delta_{3} \delta_{4}+\xi \sigma_{3} / \delta_{4}$.

The analysis of (58) is similar to that of (37), and the equilibria of (34) can be obtained as follows:

$$
N_{0}=\left(x_{0}, y_{0}, z_{0}, u_{0}, v_{0}\right)=\left(0, y_{0}, \frac{\sigma_{2}}{\delta_{2}}, \frac{\gamma_{1} \delta_{2} y_{0}+\gamma_{2} \sigma_{2}}{\delta_{2} \delta_{31}}, \frac{\sigma_{3}}{\delta_{4}}\right) \text {, }
$$

where $y_{0}=-B+\sqrt{B^{2}-4 A C} / 2 A$ with $A=\theta \gamma_{1} \delta_{2}>0$, $B=\delta_{31}\left(\delta_{1} \delta_{2}-\rho \sigma_{2}\right)+\theta \gamma_{2} \sigma_{2}$, and $C=-\sigma_{1} \delta_{2} \delta_{31}<0$.

$$
\begin{aligned}
N^{*}= & \left(x^{*}, y^{*}, z^{*}, u^{*}, v^{*}\right)=\left(x^{*}, \alpha\left(1-\beta x^{*}\right), \frac{\sigma_{2}}{\delta_{2}-\omega_{2} x^{*}},\right. \\
& \left.\frac{\alpha \gamma_{1}\left(1-\beta x^{*}\right)\left(\delta_{2}-\omega_{2} x^{*}\right)+\gamma_{2} \sigma_{2}}{\delta_{31}\left(\delta_{2}-\omega_{2} x^{*}\right)}, \frac{\sigma_{3}}{\delta_{4}}\right),
\end{aligned}
$$

where $x^{*} \in(0, \mu)$ and satisfies the equation $c_{3} x^{3}+c_{2} x^{2}+$ $c_{1} x+c_{0}=0$ with coefficients

$$
\begin{aligned}
c_{3}= & \theta \alpha^{2} \beta^{2} \gamma_{1} \omega_{2}>0, \\
c_{2}= & -\theta \alpha^{2} \beta \gamma_{1} \omega_{2}-\alpha \beta\left[\theta \alpha \gamma_{1}\left(\beta \delta_{2}+\omega_{2}\right)+\delta_{1} \delta_{31} \omega_{2}\right]<0, \\
c_{1}= & \theta \alpha\left(2 \alpha \beta \gamma_{1} \delta_{2}+\alpha \gamma_{1} \omega_{2}+\beta \gamma_{2} \sigma_{2}\right)+\alpha \delta_{31}\left[\delta_{1} \omega_{2}\right. \\
& \left.-\beta\left(\rho \sigma_{2}-\delta_{1} \delta_{2}\right)\right]-\sigma_{1} \omega_{2} \delta_{31}, \\
c_{0}= & \sigma_{1} \delta_{2} \delta_{31}+\alpha \delta_{31}\left(\rho \sigma_{2}-\delta_{1} \delta_{2}\right)-\theta \alpha\left(\alpha \gamma_{1} \delta_{2}+\gamma_{2} \sigma_{2}\right) .
\end{aligned}
$$

For system (34) at $N_{0}=\left(x_{0}, y_{0}, z_{0}, u_{0}, v_{0}\right)$, Jacobian matrix is expressed as follows:

$$
\mathbf{J}\left(N_{0}\right)=\left(\begin{array}{ccccc}
\alpha-y_{0} & 0 & 0 & 0 & 0 \\
0 & -\frac{\sigma_{1}}{y_{0}} & \rho y_{0} & -\theta y_{0} & 0 \\
\omega_{2} z_{0} & 0 & -\delta_{2} & 0 & 0 \\
0 & \gamma_{1} & \gamma_{2} & -\delta_{31} & \xi u_{0} \\
0 & 0 & 0 & 0 & -\delta_{4}
\end{array}\right) .
$$

The corresponding characteristic equation is

$$
\left|\mathbf{J}\left(N_{0}\right)-\lambda \mathbf{I}\right|=\left(\lambda+\delta_{4}\right)\left|\mathbf{J}\left(E_{0}\right)-\lambda \mathbf{I}\right|=0 .
$$

Thus, the stability analysis of $N_{0}$ is similar to that of $E_{0}$. Set $\theta_{8}=\alpha \delta_{31}\left(\rho \sigma_{2}-\delta_{1} \delta_{2}\right)+\sigma_{1} \delta_{2} \delta_{31} / \alpha\left(\alpha \gamma_{1} \delta_{2}+\sigma_{2} \gamma_{2}\right)$, and we can get the following result.

Theorem 11. System (34) always has one tumor-free equilibrium $N_{0}$, which is LAS if the inequality $\theta<\theta_{8}$ holds.

For system (34) at $N^{*}=\left(x^{*}, y^{*}, z^{*}, u^{*}, v^{*}\right)$, Jacobian matrix is given as follows:

$$
\mathbf{J}\left(N^{*}\right)=\left(\begin{array}{ccccc}
-\alpha \beta x^{*} & -x^{*} & 0 & 0 & 0 \\
0 & -\frac{\sigma_{1}}{y^{*}} & \rho y^{*} & -\theta y^{*} & 0 \\
\omega_{2} z^{*} & 0 & \omega_{2} x^{*}-\delta_{2} & 0 & 0 \\
0 & \gamma_{1} & \gamma_{2} & -\delta_{31} & \xi u^{*} \\
0 & 0 & 0 & 0 & -\delta_{4}
\end{array}\right) .
$$

The corresponding characteristic equation is

$$
\left|\mathbf{J}\left(N^{*}\right)-\lambda \mathbf{I}\right|=\left(\lambda+\delta_{4}\right)\left|\mathbf{J}\left(E^{*}\right)-\lambda \mathbf{I}\right|=0 .
$$

Thus, the stability analysis of $N^{*}$ is similar to that of $E^{*}$. Set $\theta_{9}=\rho \delta_{31} / \gamma_{2}$ and substitute $\delta_{31}$ for $\delta_{3}$ in (47) to get new conditions: 


$$
\begin{aligned}
(h 4): B_{4}= & \alpha \beta x^{*} \frac{\sigma_{2}}{z^{*}}\left(\theta \gamma_{1} y^{*}+\delta_{31} \frac{\sigma_{1}}{y^{*}}\right) \\
& +\left(\rho \delta_{31}-\theta \gamma_{2}\right) \omega_{2} x^{*} y^{*} z^{*}>0,
\end{aligned}
$$

(h5): $B_{1} B_{2}-B_{3}=k_{1} \theta+k_{2}>0$,

(h6): $B_{1}\left(B_{2} B_{3}-B_{1} B_{4}\right)-B_{3}^{2}=B_{3}\left(k_{1} \theta+k_{2}\right)-B_{1}^{2} B_{4}$

$$
=k_{3} \theta^{2}+k_{4} \theta+k_{5}>0 \text {, }
$$

where

$$
\begin{aligned}
k_{1}= & \left(\sigma_{1}+\delta_{31} y^{*}\right) \gamma_{1}, \\
k_{2}= & \left(\delta_{31}+\frac{\sigma_{1}}{y^{*}}+\frac{\sigma_{2}}{z^{*}}\right)\left[\alpha^{2} \beta^{2} x^{* 2}+\left(\alpha \beta x^{*}+\frac{\sigma_{2}}{z^{*}}\right)\left(\frac{\sigma_{1}}{y^{*}}+\delta_{31}\right)\right. \\
& \left.+\alpha \beta x^{*} \frac{\sigma_{2}}{z^{*}}\right]+\delta_{31} \frac{\sigma_{1}}{y^{*}}\left(\frac{\sigma_{1}}{y^{*}}+\delta_{31}\right)-\rho \omega_{2} x^{*} y^{*} z^{*}, \\
k_{3}= & k_{1}\left(\alpha \beta x^{*}+\frac{\sigma_{2}}{z^{*}}\right) \gamma_{1} y^{*}, \\
k_{4}= & {\left[\left(k_{2} \gamma_{1} y^{*}+k_{1} \frac{\sigma_{1} \delta_{31}}{y^{*}}\right)\left(\alpha \beta x^{*}+\frac{\sigma_{2}}{z^{*}}\right)\right] } \\
& +k_{1}\left[\alpha \beta x^{*} \frac{\sigma_{2}}{z^{*}}\left(\delta_{31}+\frac{\sigma_{1}}{y^{*}}-\left(\alpha \beta x^{*}+\delta_{31}+\frac{\sigma_{1}}{y^{*}}+\frac{\sigma_{2}}{z^{*}}\right)^{2} \gamma_{1} y^{*}\right)\right. \\
& \left.+\left(\rho+\gamma_{2}\right) \omega_{2} x^{*} y^{*} z^{*}\right], k_{5}=k_{2}\left(\alpha \beta x^{*}+\frac{\sigma_{2}}{z^{*}}\right) \frac{\sigma_{1} \delta_{31}}{y^{*}} \\
& -\alpha \beta x^{*} \frac{\sigma_{2}}{z^{*}}\left[k_{2}\left(\delta_{31}+\frac{\sigma_{1}}{y^{*}}\right)-\left(\alpha \beta x^{*}+\delta_{31}+\frac{\sigma_{1}}{y^{*}}+\frac{\sigma_{2}}{z^{*}}\right)^{2} \frac{\sigma_{1} \delta_{31}}{y^{*}}\right] \\
& +\left(k_{2}-\delta_{31}\right) \rho \omega_{2} x^{*} y^{*} z^{*} .
\end{aligned}
$$

Therefore, we can obtain the following result.

Theorem 12. System (34) has a coexistence equilibrium $N^{*}$ when $\omega_{2}>\beta \delta_{2}, \sigma_{1} \geq \alpha\left(\alpha \rho \gamma_{1}+\delta_{1} \gamma_{2}\right) / \gamma_{2}, \theta_{9}<\theta<\theta_{8}$ or $\omega_{2}>\beta \delta_{2}$, $\sigma_{1} \leq \alpha\left(\alpha \rho \gamma_{1}+\delta_{1} \gamma_{2}\right) / \gamma_{2}, \theta_{8}<\theta<\theta_{9}$ or $\omega_{2}<\beta \delta_{2}, \theta>\theta_{8}$ hold. Further $N^{*}$ is LAS when condition (66) is satisfied.

\subsection{Numerical Simulations}

3.2.1. ACI Model $\left(\sigma_{1}>0, \sigma_{3}=0\right)$. We conduct numerical simulations of the ACI model. We choose $\omega_{2}=0.0004$ and the other parameter values are given in (22). We find the stability region of $E^{*}=\left(x^{*}, y^{*}, z^{*}, u^{*}\right)$, as shown in Figure 5(a). $E^{*}$ is stable in region I and unstable in region II.

Case (a): we choose a point $G_{1}=\left(\sigma_{1}, \theta\right)=(0.4,0.5)$ in the region $\mathbf{I}$, then system (34) has one coexistence equilibrium:

$$
E^{*}=(11.560,1.598,7.543,6.993) .
$$

The eigenvalues of characteristic equation of (47) are $-0.269,-0.041,-0.1388+0.334 i$ and $-0.1388-0.334 i$,

so $E^{*}$ is stable, as shown in Figure 6(b).

Case (b): we choose a point $G_{2}=\left(\sigma_{1}, \theta\right)=(0.4,0.55)$ in the region II; then, system (34) has one coexistence equilibrium:

$$
E^{*}=(69.131,1.409,13.895,11.962)
$$

The eigenvalues of characteristic equation of (47) are $-0.830,-0.021,0.0326+0.6039 i$ and $0.0326-0.6039 i$,

so $E^{*}$ is unstable, as shown in Figures 6(c) and 6(d). According to the stability condition of tumor-free equilibrium $E_{0}$ in Theorem 5, ACI curve can be obtained as

$\theta=\frac{\alpha \delta_{3}\left(\rho \sigma_{2}-\delta_{1} \delta_{2}\right)+\sigma_{1} \delta_{2} \delta_{3}}{\alpha\left(\alpha \gamma_{1} \delta_{2}+\gamma_{2} \sigma_{2}\right)}=0.09391 \sigma_{1}+0.452$

We find the stability region of $E_{0}=\left(0, y_{0}, z_{0}, u_{0}\right)$ shown in Figure $5(\mathrm{~b}) . E_{0}$ is stable in the region III and unstable in the region IV. With the increase of $\sigma_{1}$, region III gradually increases, while region IV gradually decreases.

Case (c): we choose a point $G_{3}=\left(\sigma_{1}, \theta\right)=(0.4,0.48)$ in the region III; then, system (34) has a tumor-free equilibrium:

$$
E_{0}=(0,1.782,6.909,6.596) .
$$

The eigenvalues of Jacobian matrix of $E_{0}$ are

$-0.055,-0.146,-0.237+0.358 i$ and $-0.237-0.358 i$,

so $E_{0}$ is stable, as shown in Figure 6(a).

Case (d): we choose a point $G_{4}=\left(\sigma_{1}, \theta\right)=(0.4,0.53)$ in the region IV; then, system (34) has a tumor-free equilibrium:

$$
E_{0}=(0,1.141,6.909,6.212) .
$$

The eigenvalues of Jacobian matrix of $E_{0}$ are

$0.494,-0.055,-0.3+0.297 i$ and $-0.3-0.297 i$,

so $E_{0}$ is unstable.

We choose ACI parameter $\sigma_{1}=0.4$ to study the relationship between the number of TCs $x$ and the parameter $\theta$ (see Figure 5(c)). By some calculations, we have $\theta_{3}=\rho \delta_{3} / \gamma_{2}=$ 


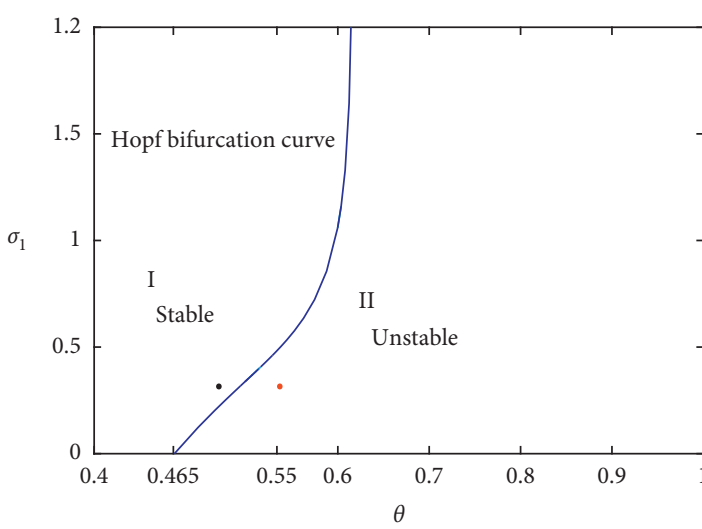

(a)

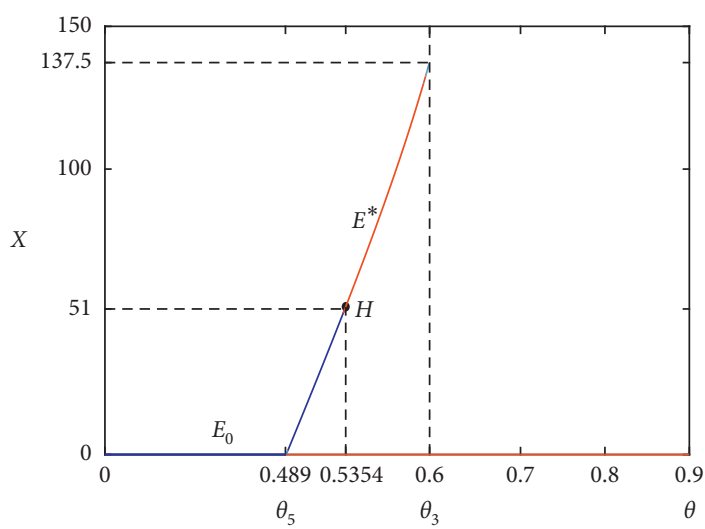

(c)

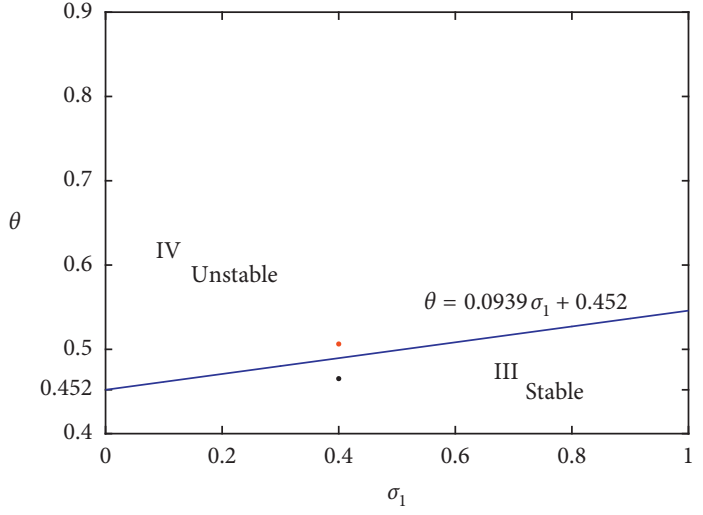

(b)

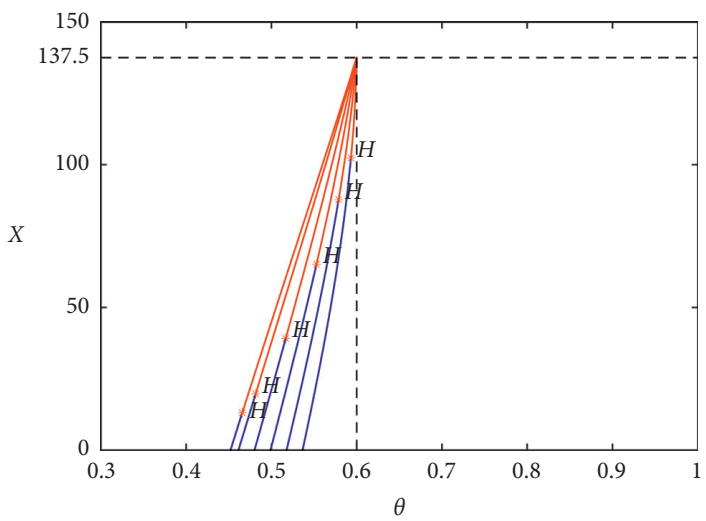

(d)

FiguRE 5: (a) Stability region of $E^{*}$ and Hopf bifurcation curve in $\theta-\sigma_{1}$ parameter plane. $E^{*}$ is stable in region I and unstable in region II. (b) ACI curve. $E_{0}$ is stable in region III and unstable in region IV. (c) The bifurcation diagram of $x$ with respect to $\theta$ for fixed $\sigma_{1}=0.4$. Hopf bifurcation point appears at $\theta_{A C I}^{*}=0.5354$. (d) The bifurcation diagram of $x$ with respect to $\theta$ for different $\sigma_{1}=0,0.1,0.3,0.5,0.7,0.9$ (the blue curves represent the stable steady states of system (34), while the red curves represent the unstable steady states).

0.6, $\theta_{5}=\alpha \delta_{3}\left(\rho \sigma_{2}-\delta_{1} \delta_{2}\right)+\sigma_{1} \delta_{2} \delta_{3} / \alpha\left(\alpha \gamma_{1} \delta_{2}+\gamma_{2} \sigma_{2}\right)=0.489$. And Hopf bifurcation point appears at $\theta_{A C I}^{*}=0.5354$. We can obtain the following result.

Proposition 5. When $0<\theta<\theta_{5}, E_{0}$ exists and is LAS, and $E^{*}$ is nonexistent. When $\theta_{5}<\theta<\theta_{A C I}^{*}, E_{0}$ exists and is unstable, and $E^{*}$ exists and is LAS. When $\theta_{A C I}^{*}<\theta<\theta_{3}, E_{0}$ exists and is unstable, and $E^{*}$ exists and is unstable. When $\theta>\theta_{3}, E_{0}$ exists and is unstable, and $E^{*}$ is nonexistent.

Next, we choose different ACI parameters $\sigma_{1}=0,0.1,0.3,0.5,0.7,0.9$, to study the relationship between the number of TCs $x$ and the parameter $\theta$ (see Figure 5(d)).

By comparing the curves in Figure 5(d), we see that, as $\sigma_{1}$ increases gradually, the stable region of tumor-free equilibrium $E_{0}$ of system (34) gradually increases (the intersection point of the curve and the $x$-coordinate gradually moves to the right) and the stability region of equilibrium $E^{*}$ in system (34) gradually increases (the blue curve gradually moves upward). This indicates that increasing the injection volume of adoptive $T$ cells can not only delay the inhibitory effect of Tregs on tumor immune response but also help the immune system to remove more TCs. It also helps the immune system to control more TCs, keeping them at a stable state.

3.2.2. MAI Model $\left(\sigma_{1}=0, \sigma_{3}>0\right)$. We conduct numerical simulations of the MAI model. We choose $\omega_{2}=0.0004, \xi=$ 0.05 , and $\delta_{4}=0.25$ and the other parameter values are given in (22). We find the stability region of $F^{*}=\left(x^{*}, y^{*}, z^{*}, u^{*}\right)$, as shown in Figure $7(\mathrm{a}) . F^{*}$ is stable in region $\mathbf{I}$ and unstable in region II.

Case (a): we choose a point $M_{1}=\left(\sigma_{3}, \theta\right)=(0.4,0.6)$ in region I; then, system (34) has one coexistence equilibrium:

$$
F^{*}=(2.413,1.628,7.032,5.002,1.6)
$$

The eigenvalues of Jacobian matrix of $F^{*}$ are

$$
\begin{aligned}
& -0.25,-0.146+0.336 i,-0.146-0.336 i,-0.0499 \\
& +0.033 i \text { and }-0.0499-0.033 i,
\end{aligned}
$$




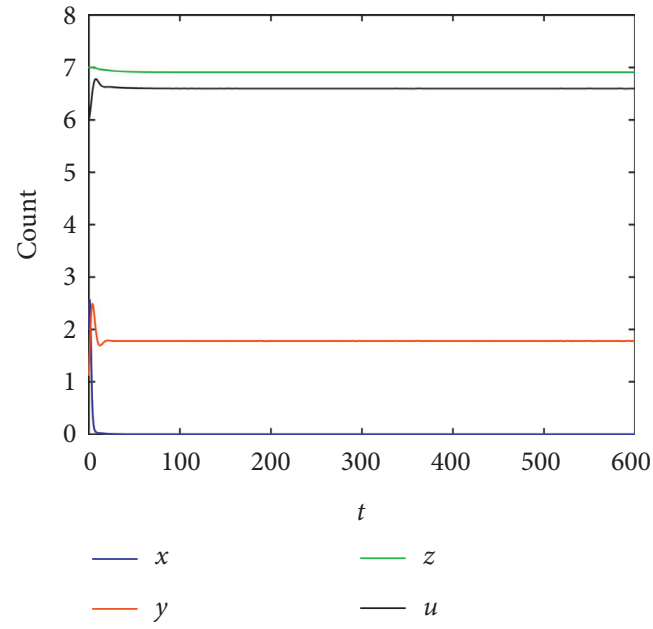

(a)

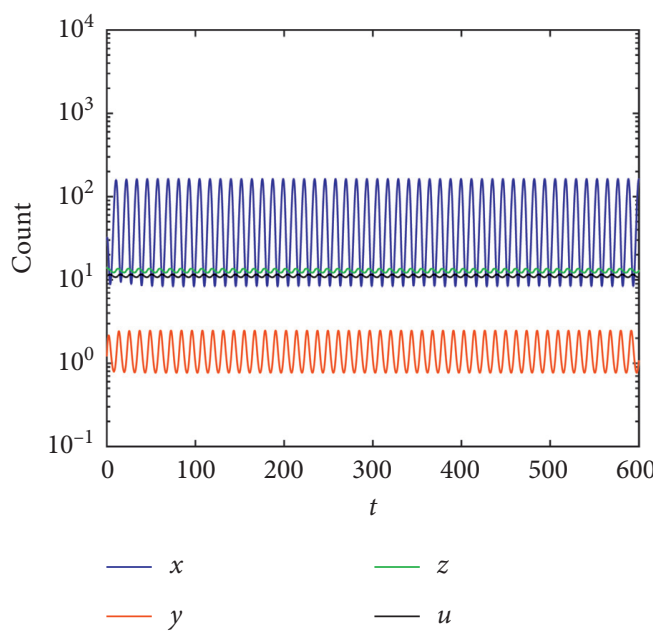

(c)

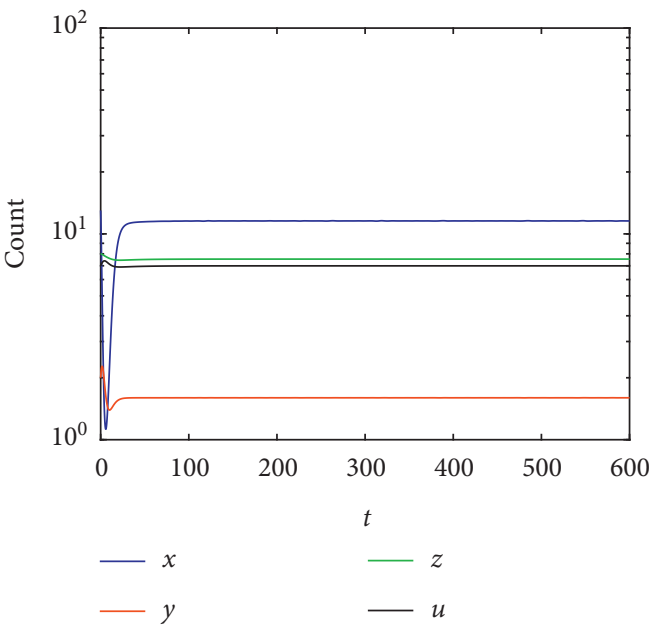

(b)

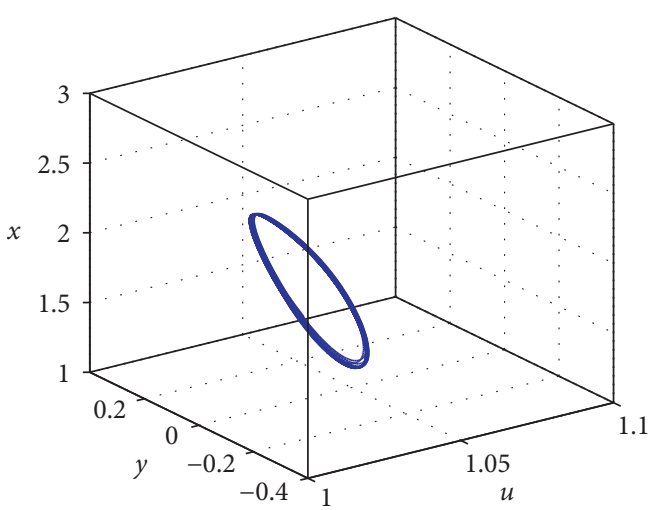

(d)

Figure 6: Choose $\sigma_{1}=0.4$, and the other parameter values are given in (22). (a) When $\theta=0.48, E_{0}(0,1.782,6.909,6.596)$ is LAS. (b) When $\theta=0.50, E^{*}(11.560,1.598,7.543,6.993)$ is LAS. (c) When $\theta=0.55, E^{*}(69.131,1.409,13.895,11.962)$ is unstable. (d) When $\theta=0.55$, the 3D phase portrait depicts TCs, ECs, and Tregs.

so $F^{*}$ is stable, as shown in Figure $8(\mathrm{~b})$.

Case (b): we choose a point $M_{2}=\left(\sigma_{3}, \theta\right)=(0.4,0.635)$ in region $\mathbf{I I}$; then, system (34) has one coexistence equilibrium:

$$
F^{*}=(27.345,1.546,8.624,5.93,1.6) \text {. }
$$

The eigenvalues of Jacobian matrix of $F^{*}$ are

$$
\begin{gathered}
-0.4334,-0.25,-0.0644,0.0172 \\
+0.429 i \text { and } 0.0172-0.429 i,
\end{gathered}
$$

so $F^{*}$ is unstable, as shown in Figures $8(\mathrm{c})$ and $8(\mathrm{~d})$. According to the stability condition of tumor-free equilibrium $F_{1}$ in Theorem 7 , MAI curve can be obtained as $\theta=\left(\delta_{3} \delta_{4}+\xi \sigma_{3}\right)\left(\rho \sigma_{2}-\delta_{1} \delta_{2}\right) /\left(\alpha \gamma_{1} \delta_{2}+\sigma_{2} \gamma_{2}\right)$

$\delta_{4}=0.3616 \sigma_{3}+0.452$.

We find the stability region of $F_{1}$, as shown in Figure $7(\mathrm{~b}) . F_{0}$ is stable in region III and unstable in region IV. With the increase of $\sigma_{3}$, region III gradually increases, while region IV gradually decreases.

Case (c): we choose a point $M_{3}=\left(\sigma_{3}, \theta\right)=(0.4,0.5)$ in region III; then, system (34) has a tumor-free equilibrium:

$$
F_{1}=(0,2.556,6.909,5.349,1.6)
$$

The eigenvalues of Jacobian matrix of $F_{1}$ are 


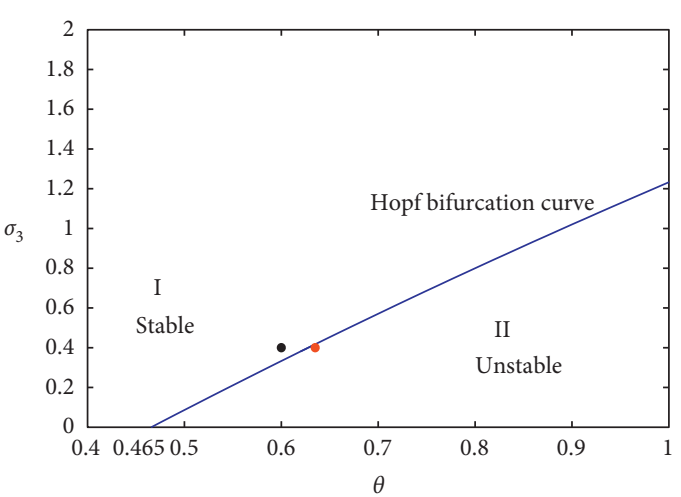

(a)

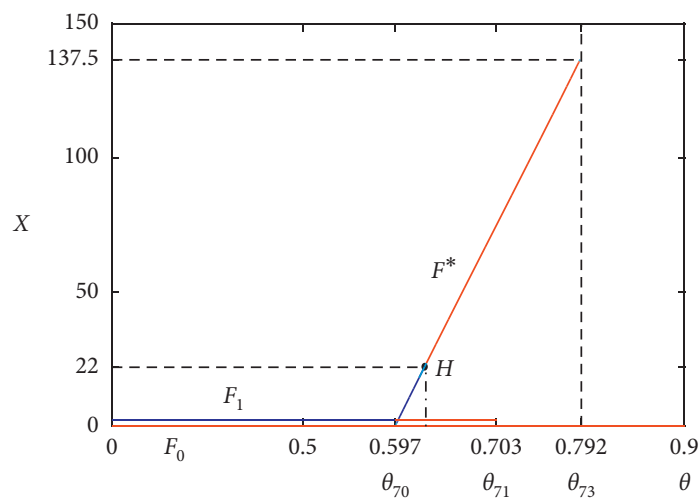

(c)

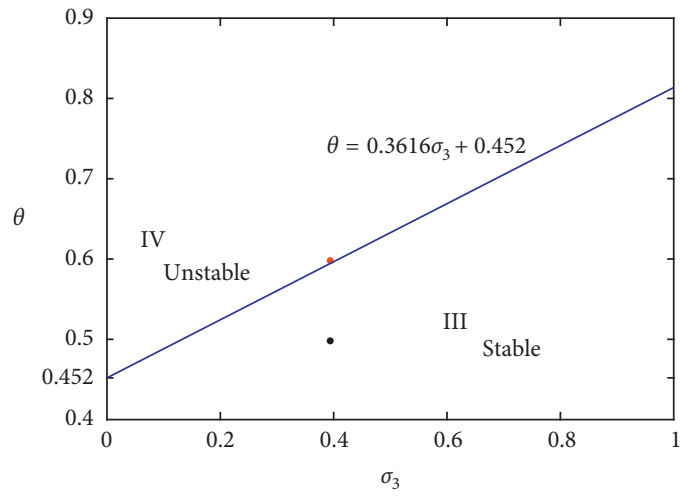

(b)

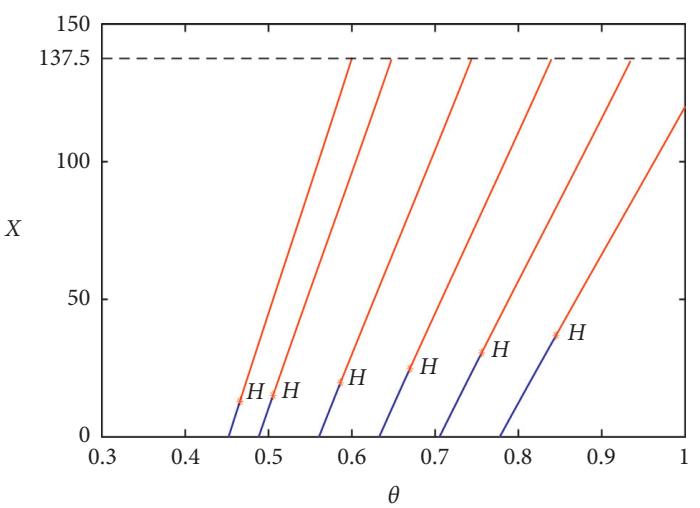

(d)

FIGURE 7: (a) Stability region of $F^{*}$ and Hopf bifurcation curve in $\theta-\sigma_{3}$ parameter plane. $F^{*}$ is stable in region I and unstable in region II. (b) MAI curve. $F_{1}$ is stable in region III and unstable in region IV. (c) The bifurcation diagram of $x$ with respect to $\theta$ for fixed $\sigma_{3}=0.4$. Hopf bifurcation point appears at $\theta_{M A I}^{*}=0.627$. (d) The bifurcation diagrams of $x$ with respect to $\theta$ for different $\sigma_{3}=0,0.1,0.3,0.5,0.7,0.9$ (the blue curves represent the stable steady states of system (34), while the red curves represent the unstable steady states).

$-0.92,-0.25,-0.055,-0.164+0.428 i$ and $-0.164-0.428 i$,

so $F_{1}$ is stable, as shown in Figure $8(a)$.

Case (d): we choose a point $M_{4}=\left(\sigma_{3}, \theta\right)=(0.4,0.6)$ in region IV; then, system (34) has a tumor-free equilibrium:

$$
F_{1}=(0,1.575,6.909,4.903,1.6) .
$$

The eigenvalues of Jacobian matrix of $F_{1}$ are $0.061,-0.25,-0.055,-0.164+0.338 i$ and $-0.164-0.338 i$,

so $F_{1}$ is unstable.

We choose MAI parameter $\sigma_{3}=0.4$ to study the relationship between the number of TCs $x$ and the parameter $\theta$ (see Figure $7(\mathrm{c})$ ). By some calculations, we have $\theta_{70}=\delta_{31}\left(\rho \sigma_{2}-\delta_{1} \delta_{2}\right) / \alpha \gamma_{1} \delta_{2}+\gamma_{2} \sigma_{2}=0.597, \theta_{71}=\delta_{31}\left(\rho \sigma_{2}-\right.$ $\left.\delta_{1} \delta_{2}\right) / \gamma_{2} \sigma_{2}=0.703$, and $\theta_{73}=\rho \delta_{31} / \gamma_{2}=0.792$. And Hopf bifurcation point appears at $\theta_{M A I}^{*}=0.627$. We can obtain the following result.

Proposition 6. When $0<\theta<\theta_{70}, F_{0}$ exists and is unstable, $F_{1}$ exists and is LAS, and $F^{*}$ exists and is LAS. When
$\theta_{70}<\theta<\theta_{M A I}^{*}, F_{0}$ exists and is unstable, $F_{1}$ exists and is unstable, and $F^{*}$ exists and is unstable. When $\theta_{M A I}^{*}<\theta<\theta_{71}, F_{0}$ exists and is unstable, $F_{1}$ exists and is unstable, and $F^{*}$ exists and is unstable. When $\theta_{71}<\theta<\theta_{73}, F_{0}$ exists and is unstable, $F_{1}$ is nonexistent, and $F^{*}$ exists and is unstable. When $\theta>\theta_{3}, F_{0}$ exists and is unstable, $F_{1}$ is nonexistent, and $F^{*}$ is nonexistent.

Next, we choose different MAI parameters $\sigma_{3}=0,0.1,0.3,0.5,0.7,0.9$, to study the relationship between the number of TCs $x$ and the parameter $\theta$ (see Figure $7(d)$ ).

By comparing the curves in Figure $7(d)$, we can find that, with the gradual increase of $\sigma_{3}$, the stability region of the tumour-free equilibrium $F_{1}$ of system (34) gradually increases (the intersection point of curves and $x$-coordinate gradually moves to the right). This shows that the increase of antibody injection quantity can help to slow down Tregs inhibition of tumor immune responses. The stability region of equilibrium $F^{*}$ of system (34) gradually increases (the blue curve gradually moves upward), which means that increasing the amount of antibody injected can help the immune system to control more TCs. By comparing Figures 5 and 7, we find that the effect of MAI is better than that of AIC in delaying the inhibitory effect of Tregs on tumor immune response (at the same injection dose, the intersection point of curves and $x$-coordinate in Figure 7 moves to the right more widely than that in Figure 5). AIC is more effective than MAI in controlling TCs (the blue 


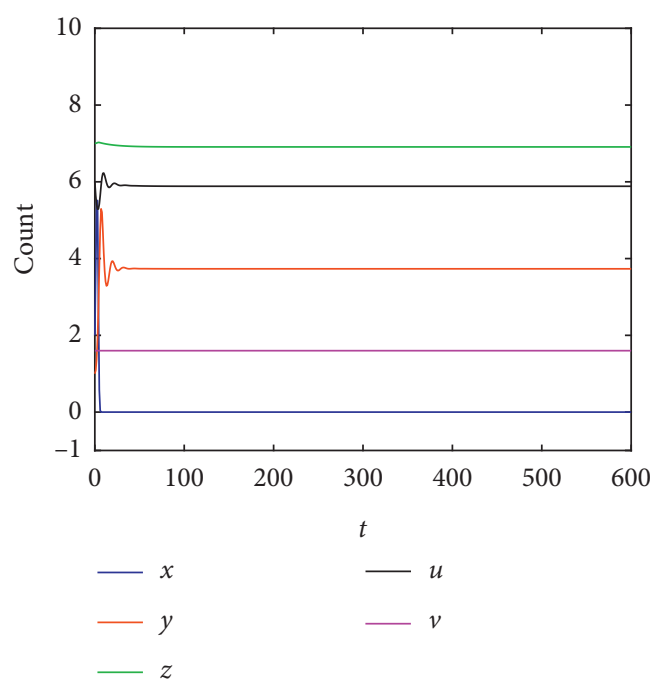

(a)

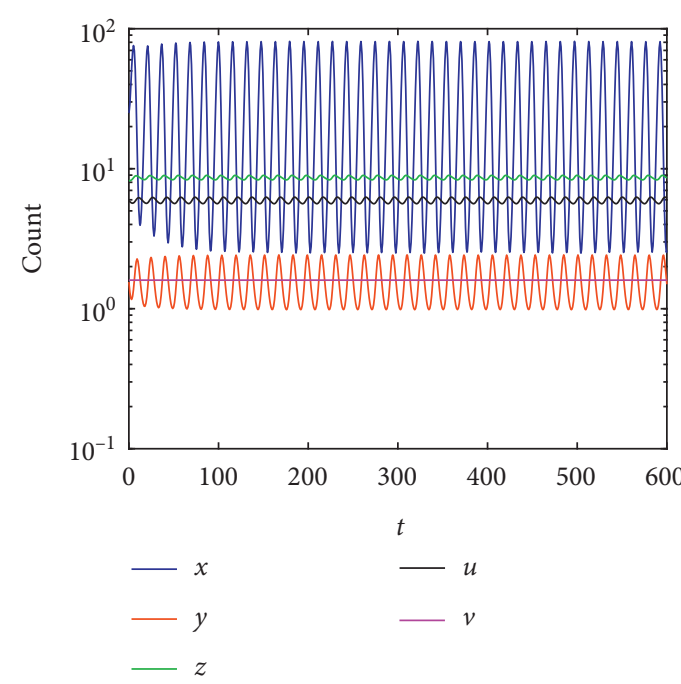

(c)

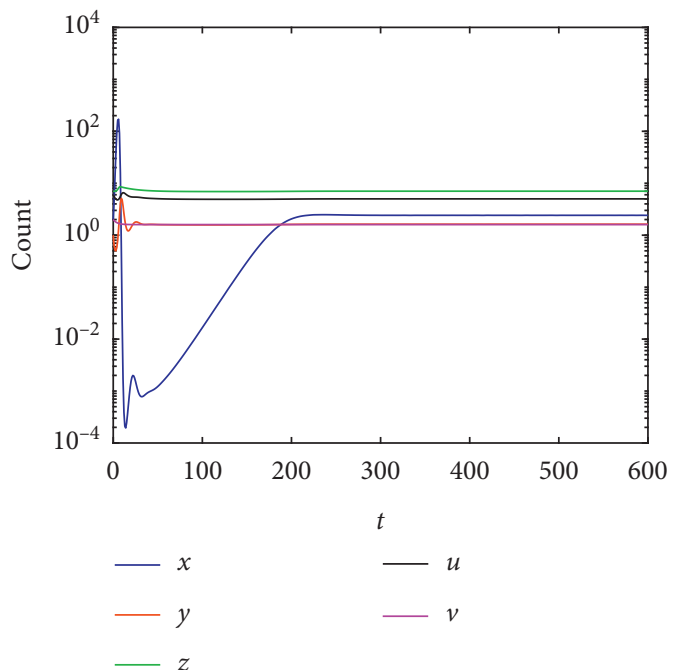

(b)

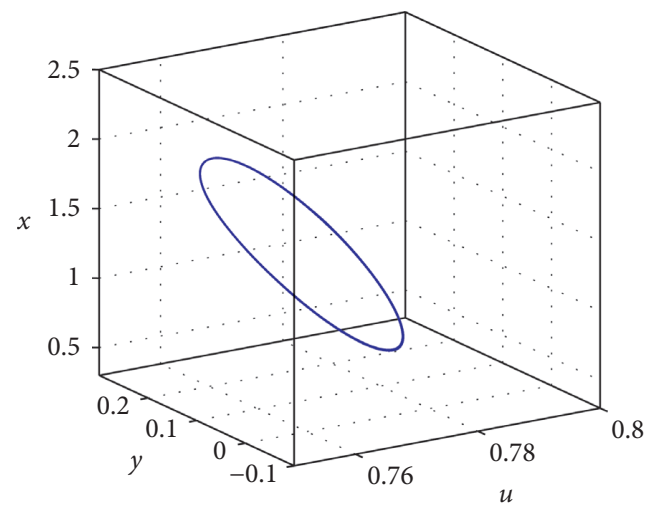

(d)

Figure 8: Choose $\sigma_{3}=0.4$, and the other parameter values are given in $(22)$. (a) When $\theta=0.5, F_{1}(0,2.556,6.909,5.349,1.6)$ is LAS. (b) When $\theta=0.6, F^{*}(2.413,1.628,7.032,5.002,1.6)$ is LAS. (c) When $\theta=0.635, F^{*}(27.345,1.546,8.624,5.93,1.6)$ is unstable. (d) When $\theta=0.635$, the $3 \mathrm{D}$ phase portrait depicts TCs, ECs, and Treg.

curve in Figure 7 moves up more than the blue curves in Figure 5 at the same injection dose).

3.2.3. Combined Immunotherapy Model $\left(\sigma_{1}>0, \sigma_{3}>0\right)$. We conduct numerical simulations of the combined immunotherapy model. We choose $\omega_{2}=0.0004$, $\xi=0.05$, and $\delta_{4}=0.25$, and the other parameter values are given in (22). We find the stability region of $N^{*}=\left(x^{*}, y^{*}\right.$, $\left.z^{*}, u^{*}, v^{*}\right)$, as shown in Figure 9(a). When $\sigma_{3}$ increases gradually, the stability region of $N^{*}$ also increases gradually.

Case (a): we choose a point $L_{1}=\left(\theta, \sigma_{1}\right)=(0.7,0.4)$ on the left of the curve $\sigma_{3}=0.5$; then, system (34) has one coexistence equilibrium:

$$
N^{*}=(11.56,1.59,7.54,4.99,2) .
$$

The eigenvalues of Jacobian matrix of (64) are

$$
-0.06,-0.176,-0.25,-0.22-0.37 i \text { and }-0.22+0.37 i
$$

so $N^{*}$ is stable, as shown in Figure 10(b).

Case (b): we choose a point $L_{2}=\left(\theta, \sigma_{1}\right)=(0.78,0.4)$ on the right of the curve $\sigma_{3}=0.5$; then, system (34) has one coexistence equilibrium:

$$
N^{*}=(77.887,1.381,15.936,9.698,2) .
$$




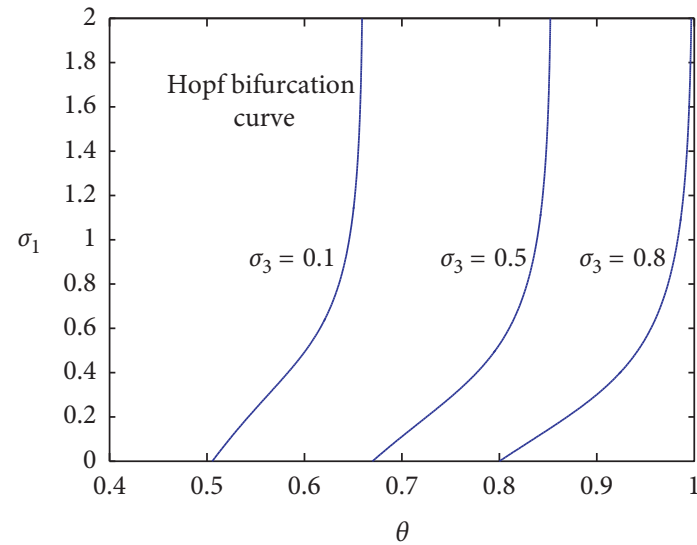

(a)

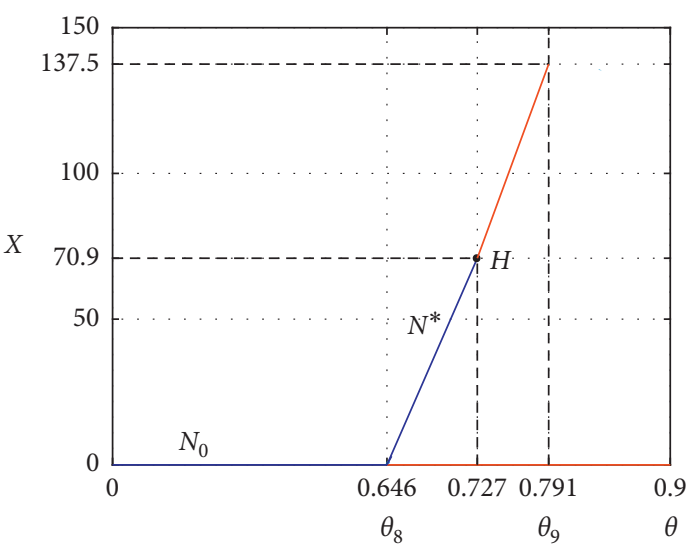

(c)

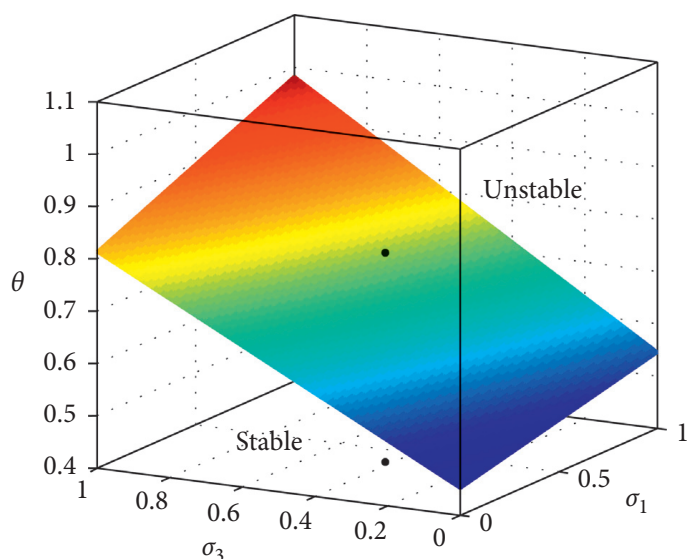

(b)

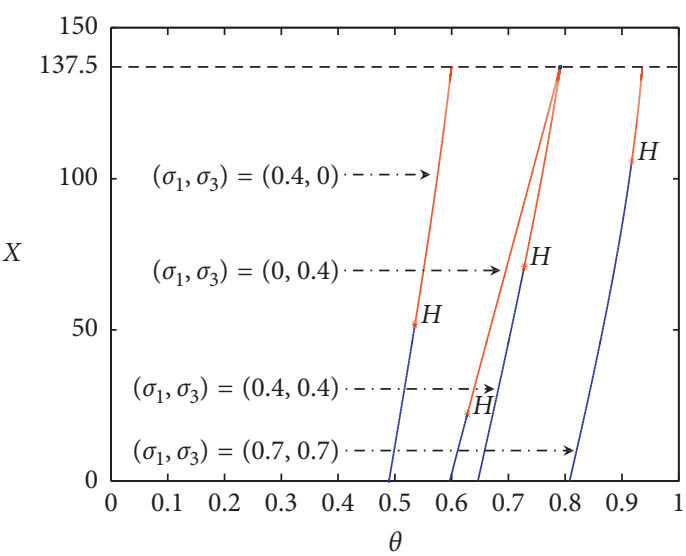

(d)

FiguRE 9: (a) Choose different $\sigma_{3}=0.1,0.5,0.8$. Stability region of $N^{*}$ and Hopf bifurcation curve in $\theta-\sigma_{1}$ parameter plane. $N^{*}$ is stable to the left of the curve and unstable to the right of the curve. (b) Combined immunotherapy surface. $N_{0}$ is stable below the surface and unstable above the surface. (c) When $\sigma_{1}=0.4$ and $\sigma_{3}=0.4$, the bifurcation diagram of $x$ with respect to $\theta$ for system (34). Hopf bifurcation point appears at $\theta_{C I}^{*}=0.727$. (d) Choose different $\left(\sigma_{1}, \sigma_{3}\right)=(0.4,0),(0,0.4),(0.4,0.4),(0.7,0.7)$. The bifurcation diagram of $x$ with respect to $\theta$ (the stable steady state is represented by the blue curve, and the unstable one corresponds to the red curve).

The eigenvalues of Jacobian matrix of (64) are

$$
\begin{aligned}
& -0.906238,-0.0248,-0.25,0.00641 \\
& -0.66 i \text { and } 0.00641+0.66 i,
\end{aligned}
$$

so $N^{*}$ is unstable, as shown in Figures 10(c) and 10(d). According to the stability condition of tumor-free equilibrium $N_{0}$ in Theorem 11, combined immunotherapy surface can be obtained as

$$
\begin{aligned}
\theta & =\frac{\alpha \delta_{31}\left(\rho \sigma_{2}-\delta_{1} \delta_{2}\right)+\sigma_{1} \delta_{2} \delta_{31}}{\alpha\left(\alpha \gamma_{1} \delta_{2}+\sigma_{2} \gamma_{2}\right)} \\
& =\left(0.055 \sigma_{1}+0.2647\right)\left(1.366 \sigma_{3}+1.707\right)
\end{aligned}
$$

Therefore, the stability region of tumor-free equilibrium $N_{0}$ of system (34) is obtained (see Figure 9(b)). Case (c): we choose a point $L_{3}=\left(\sigma_{1}, \sigma_{2}, \theta\right)=(0.5,0.5$, 0.4 ) below surface (90); then, system (34) has a tumorfree equilibrium:

$$
N_{0}=(0,8.3,6.909,7.505,2) .
$$

The eigenvalues of Jacobian matrix of (64) are $-6.664,-0.055,-0.25,-0.204+0.69 i$ and $-0.204-0.69 i$,

so $N_{0}$ is stable, as shown in Figure 10(a).

Case (d): we choose a point $L_{4}=\left(\sigma_{1}, \sigma_{2}, \theta\right)=$ $(0.5,0.5,0.8)$ above surface $(90)$; then, system (34) has a tumor-free equilibrium:

$$
N_{0}=(0,0.886,6.909,4.32,2) \text {. }
$$

The eigenvalues of Jacobian matrix of (64) are

$$
\begin{aligned}
& 0.75,-0.055,-0.25,-0.453+0.311 i \text { and } \\
& -0.453-0.311 i,
\end{aligned}
$$

so $N_{0}$ is unstable.

We choose combined immunotherapy parameter $\sigma_{1}=$ 0.4 and $\sigma_{3}=0.4$ to study the relationship between the number of TCs $x$ and the parameter $\theta$ (see Figure 9(c)). By some calculations, we have $\theta_{8}=\alpha \delta_{31}\left(\rho \sigma_{2}-\delta_{1} \delta_{2}\right)+$ 


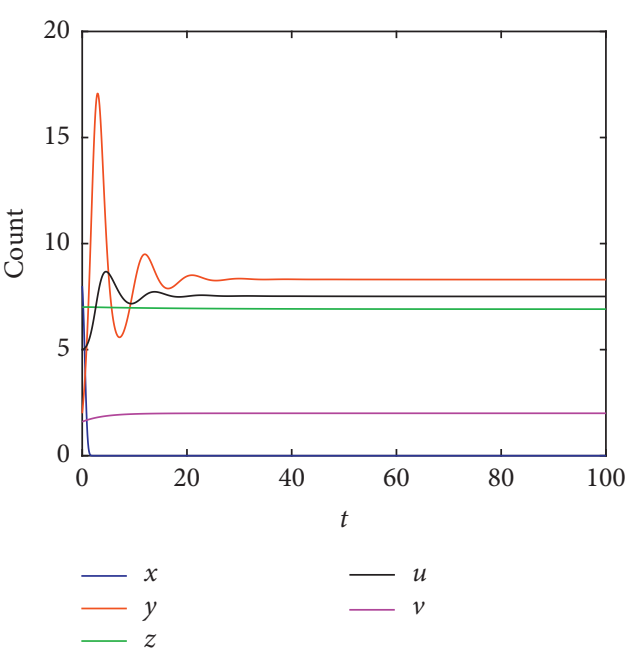

(a)

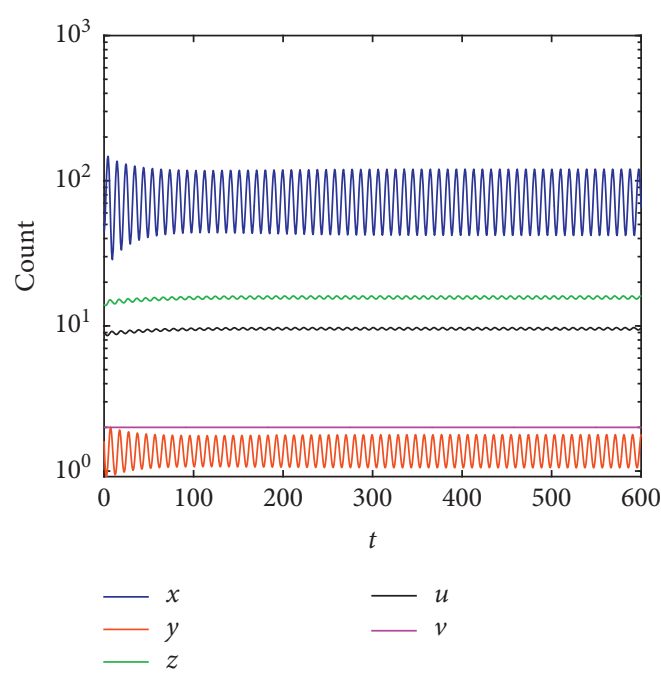

(c)

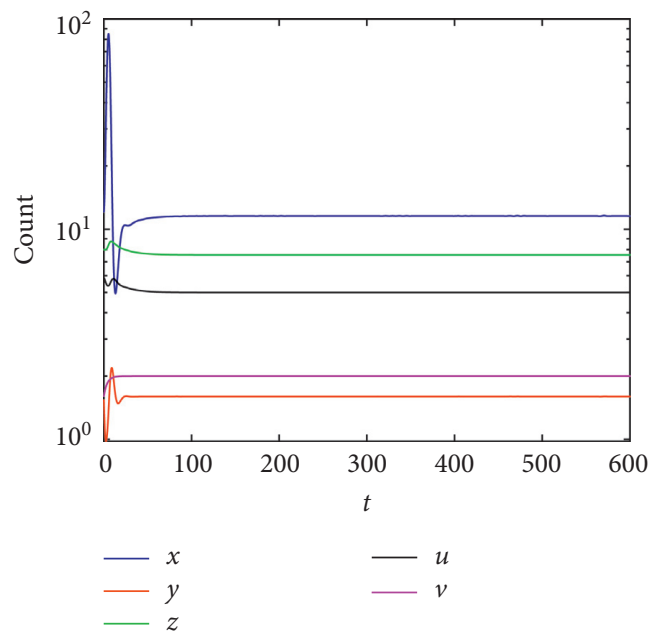

(b)

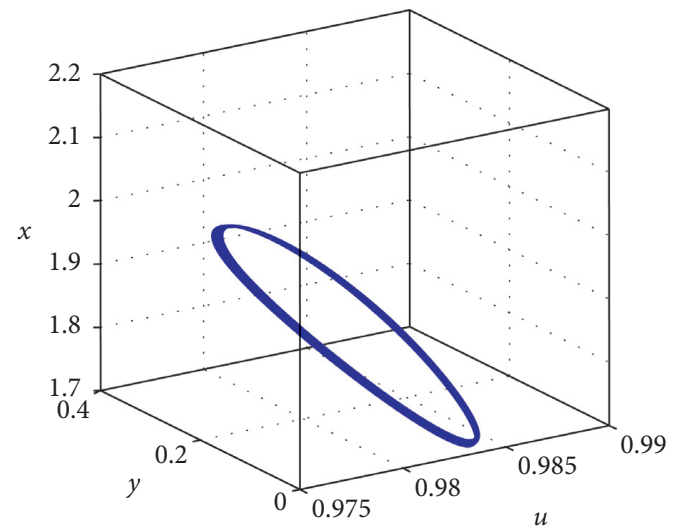

(d)

Figure 10: (a) When $\sigma_{1}=\sigma_{3}=0.5, \theta=0.4, \quad N_{0}(0,8.3,6.909,7.505,2) \quad$ is $\quad$ LAS. (b) When $\sigma_{1}=0.4, \sigma_{3}=0.5, \theta=0.7$, $N^{*}(11.56,1.59,7.54,4.99,2)$ is LAS. (c) When $\sigma_{1}=0.4, \sigma_{3}=0.5, \theta=0.78, N^{*}(77.887,1.381,15.936,9.698,2)$ is unstable. (d) When $\sigma_{1}=0.4, \sigma_{3}=0.5, \theta=0.78$, the $3 \mathrm{D}$ phase portrait depicts TCs, ECs, and Tregs.

$\sigma_{1} \delta_{2} \delta_{31} / \alpha\left(\alpha \gamma_{1} \delta_{2}+\sigma_{2} \gamma_{2}\right)=0.646$, and $\theta_{9}=\rho \delta_{31} / \gamma_{2}=0.791$ And Hopf bifurcation point appears at $\theta_{C I}^{*}=0.727$. We can obtain the following result.

Proposition 7. When $0<\theta<\theta_{8}, N_{0}$ exists and is LAS, and $N^{*}$ is nonexistent. When $\theta_{8}<\theta<\theta_{C I}^{*}, N_{0}$ exists and is unstable, and $N^{*}$ exists and is LAS. When $\theta_{C I}^{*}<\theta<\theta_{9}, N_{0}$ exists and is unstable, and $N^{*}$ exists and is unstable. When $\theta>\theta_{9}$, $N_{0}$ exists and is unstable, and $N^{*}$ is nonexistent.

For combined immunotherapy, by comparing the curves in Figure $9(d)$, we know that, with the increase of $\sigma_{1}$ and $\sigma_{3}$, the stability region of the tumour-free equilibrium of $N_{0}$ of system (34) gradually increases (the intersection point of curves and $x$-coordinate gradually moves to the right), and the stability region of equilibrium $N^{*}$ of system (34) gradually increases (the blue curve gradually moves upward). By comparing Figure 9 with Figures 5 and 7, it can be seen that combined immunotherapy has better effects on delaying the inhibitory effect of Tregs on tumor immune response and helps the immune system to control more TCs than ACI or MAI.

\section{Discussion and Conclusion}

Tregs-mediated tumor immune escape is one of the core mechanisms of tumor immune regulation. And Tregs have been found to mediate tumor evasion and immune escape in many different solid tumors [46]. The study on Tregs has a very high research value and application prospect in the immunotherapy of tumors. If the activity of Tregs is controlled or blocked during the tumor immune response, or a barrier is set to prevent Tregs from migrating into the tumor microenvironment, then the effect of tumor immunotherapy can be improved [47]. 
First, we developed a mathematical model to study the inhibitory role of Tregs in the tumor immune system. For the lower recognition of tumor antigens by the immune system, the stronger the inhibition effect of Tregs on ECs, TCs can easily escape the control of the immune system (see Figure 4(a)). When the immune system is highly sensitive to tumor antigens, the immune system activates ECs; the stronger the inhibition effect of Tregs on ECs, the more complicated interactions between TCs and immune cells (see Figure 4(b)).

Second, we incorporated the previous mathematical model with three types of immunotherapy to obtain ACI model, MAI model, and combined both ACI and MAI model. Through the theoretical analysis and numerical simulations, we found that ACI can control more TCs, but have no obvious effects on reducing the inhibitory effect of Tregs on ECs (see Figure 5). MAI can effectively reduce the inhibitory effect of Tregs on ECs, but cannot control more TCs (see Figure 7). However, combination immunotherapy with $\mathrm{ACI}$ and MAI is more effective than single immunotherapy. It can not only significantly reduce the inhibitory effect of Tregs on ECs but also help the immune system to kill TCs to the maximum extent (see Figure 9). Therefore, we recommend the use of combined immunotherapy in the treatment of tumors. Besides, clinical trials are needed to further evaluate the safety and efficacy of combined immunotherapy.

This paper focused on the general process of tumor immune response with negative feedback. Using the mathematical model, it is possible to simulate the state of tumors in the immune system at different inhibition states. The results of the study can contribute to the understanding of tumor immunity; at the same time, it also provides new ideas for the treatment of tumors. However, due to the complexity and heterogeneity of tumor microenvironment, there is still a certain gap between the mathematical model and the description of the real interactions between the tumor and immune system. Therefore, specific tumor microenvironment and heterogeneous tumoral populations should be considered in practical application to make the model more realistic [34]. In addition to the bilinear incidence model considered in this paper, nonbilinear model with saturation incidence should be employed in the further study [35]. Besides, other important factors such as immune activation delays [36-38, 48], stochastic effects [39, 40, 49], and impulsive perturbations $[41,42,50]$ can be considered in the modelling of the tumor immune system. The tumor immune response dynamics in vivo is very complex and not well understood primarily because the measurements of the necessary parameters are difficult in vivo [43].

\section{Data Availability}

No data were used to support this study.

\section{Conflicts of Interest}

The authors declare that there are no conflicts of interest regarding the publication of this paper.

\section{Acknowledgments}

This research was supported by the National Natural Science Foundation of China (nos. 11871235, 11871238, and 11901225); Natural Science Foundation of Hubei Province (no. 2019CFB189); Fundamental Research Funds for the Central Universities (no. CCNU18XJ041); Aoyama Gakuin University research grant "Ongoing Research Support;" and Japan Society for the Promotion of Science "Grand-in-Aid 20K03755."

\section{References}

[1] P. Boyle, "Measuring progress against cancer in Europe: has the 15\% decline targeted for 2000 come about?" Annals of Oncology, vol. 14, pp. 1312-1325, 2003.

[2] T. Boon and P. Van der Bruggen, "Human tumor antigens recognized by T lymphocytes," The Journal of Experimental Medicine, vol. 183, no. 3, pp. 725-729, 1996.

[3] K. E. De Visser, A. Eichten, and L. M. Coussens, "Paradoxical roles of the immune system during cancer development," Nature Reviews Cancer, vol. 6, no. 1, pp. 24-37, 2006.

[4] Y. Dong, R. Miyazaki, R. Miyazaki, and Y. Takeuchi, "Mathematical modeling on helper T cells in a tumor immune system," Discrete \& Continuous Dynamical Systems-B, vol. 19, no. 1, pp. 55-72, 2014.

[5] A. Talkington, C. Dantoin, and R. Durrett, "Ordinary differential equation models for adoptive immunotherapy," Bulletin of Mathematical Biology, vol. 9, pp. 1-25, 2017.

[6] Y.-P. Lai, C.-J. Jeng, and S.-C. Chen, "The roles of CD4+T cells in tumor immunity," ISRN Immunology, vol. 2011, pp. 1-6, 2011.

[7] T. Maj, W. Wang, J. Crespo et al., "Oxidative stress controls regulatory T cell apoptosis and suppressor activity and PD-L1blockade resistance in tumor," Nature Immunology, vol. 18, no. 12, pp. 1332-1341, 2017.

[8] S. Wilson and D. Levy, "A mathematical model of the enhancement of tumor vaccine efficacy by immunotherapy," Bulletin of Mathematical Biology, vol. 74, no. 7, pp. 1485-1500, 2012.

[9] A. Albert, M. Freedman, and A. S. Perelson, "Tumors and the immune system: the effects of a tumor growth modulator," Mathematical Biosciences, vol. 50, no. 1-2, pp. 25-58, 1980.

[10] A. d'Onofrio, "A general framework for modeling tumorimmune system competition and immunotherapy: mathematical analysis and biomedical inferences," Physica D, vol. 208, pp. 220-235, 2005.

[11] D. Kirschner and J. C. Panetta, "Modeling immunotherapy of the tumor - immune interaction," Journal of Mathematical Biology, vol. 37, no. 3, pp. 235-252, 1998.

[12] S. Amarnath, C. W. Mangus, J. C. M. Wang et al., "The PDL1PD1 Axis converts human TH1 cells into regulatory T cells," Science Translational Medicine, vol. 3, no. 111, pp. 111-120, 2011.

[13] L. Spain, S. Diem, and J. Larkin, "Management of toxicities of immune checkpoint inhibitors," Cancer Treatment Reviews, vol. 44, pp. 51-60, 2016.

[14] K. S. Peggs, S. A. Quezada, A. J. Korman, and J. P. Allison, "Principles and use of anti-CTLA4 antibody in human cancer immunotherapy," Current Opinion in Immunology, vol. 18, no. 2, pp. 206-213, 2006.

[15] A. A. Hurwitz, B. A. Foster, E. D. Kwon et al., "Combination immunotherapy of primary prostate cancer in a transgenic mouse model using CTLA-4 blockade," Cancer Research, vol. 60, no. 9, pp. 2444-2448, 2000. 
[16] J. G. Egen, M. S. Kuhns, and J. P. Allison, "CTLA-4: new insights into its biological function and use in tumor immunotherapy," Nature Immunology, vol. 3, no. 7, pp. 611-618, 2002.

[17] T. Takahashi, T. Tagami, S. Yamazaki et al., "Immunologic self-tolerance maintained by $\mathrm{Cd} 25+\mathrm{Cd} 4+$ Regulatory $\mathrm{T}$ cells constitutively expressing cytotoxic T lymphocyte-associated antigen 4," The Journal of Experimental Medicine, vol. 192, no. 2, pp. 303-310, 2000.

[18] Y.-F. Ma, C. Chen, D. Li et al., “Targeting of interleukin (IL)17A inhibits PDL1 expression in tumor cells and induces anticancer immunity in an estrogen receptor-negative murine model of breast cancer," Oncotarget, vol. 8, no. 5, pp. 76147624, 2016.

[19] K. R. Jordan, V. Borges, and M. D. Mccarter, “Abstract 1671: immunosuppressive myeloid-derived suppressor cells expressing PDL1 are increased in human melanoma tumor tissue," Cancer Research, vol. 74, p. 1671, 2014.

[20] E. Baldelli, V. Calvert, and K. A. Hodge, "Abstract 5656: quantitative measurement of PDL1 expression across tumor types using laser capture microdissection and reverse phase protein microarray," Cancer Research, vol. 77, p. 5656, 2017.

[21] E. E. West, H.-T. Jin, A.-U. Rasheed et al., "PD-L1 blockade synergizes with IL-2 therapy in reinvigorating exhausted T cells," Journal of Clinical Investigation, vol. 123, no. 6, pp. 2604-2615, 2013.

[22] L. M. Francisco, P. T. Sage, and A. H. Sharpe, "The PD-1 pathway in tolerance and autoimmunity," Immunological Reviews, vol. 236, no. 1, pp. 219-242, 2010.

[23] B. Brady, "Dramatic survival benefit with nivolumab in melanoma," Cancer Research, vol. 6, p. OF7, 2016.

[24] D. Kazandjian, D. L. Suzman, G. Blumenthal et al., "FDA approval summary: nivolumab for the treatment of metastatic non-small cell lung cancer with progression on or after platinum-based chemotherapy," The Oncologist, vol. 21, no. 5, pp. 634-642, 2016.

[25] C. Voena and R. Chiarle, "Advances in cancer immunology and caner immuntherapy," Cancer Research, vol. 21, pp. 125-133, 2016.

[26] N. V. Stepanova, "Course of the immune reaction during the development of a malignant tumour," Cancer Research, vol. 24, pp. 917-923, 1979.

[27] V. A. Kuznetsov, I. A. Makalkin, M. A. Taylor, and A. S. Perelson, "Nonlinear dynamics of immunogenic tumors: parameter estimation and global bifurcation analysis," Bulletin of Mathematical Biology, vol. 56, no. 2, pp. 295-321, 1994.

[28] O. Sotolongo-Costa, L. Morales Molina, D. Rodríguez Perez, J. C. Antoranz, and M. Chacón Reyes, "Behavior of tumors under nonstationary therapy," Physica D: Nonlinear Phenomena, vol. 178, no. 3-4, pp. 242-253, 2003.

[29] M. Galach, "Dynamics of the tumor-immune system competition-the effect of time delay," International Journal of Applied Mathematics and Computer Science, vol. 13, pp. 395-406, 2003.

[30] L. G. de Pillis, W. Gu, and A. E. Radunskaya, "Mixed immunotherapy and chemotherapy of tumors: modeling, applications and biological interpretations," Journal of Theoretical Biology, vol. 238, no. 4, pp. 841-862, 2006.

[31] S. Bunimovich-Mendrazitsky, H. Byrne, and L. Stone, "Mathematical model of pulsed immunotherapy for superficial bladder cancer," Bulletin of Mathematical Biology, vol. 70, no. 7, pp. 2055-2076, 2008.

[32] A. Radunskaya, R. Kim, and T. Woods, "Mathematical modeling of tumor immune interactions: a closer look at the role of a PD-L1 inhibitor in cancer immunotherapy," Spora: $A$ Journal of Biomathematics, vol. 4, pp. 25-41, 2018.

[33] Y. Shu, J. Huang, Y. Dong, and Y. Takeuchi, "Mathematical modeling and bifurcation analysis of pro- and anti-tumor macrophages," Applied Mathematical Modelling, vol. 88, pp. 758-773, 2020.

[34] E. Piretto, M. Delitala, and M. Ferraro, "How combination therapies shape drug resistance in heterogeneous tumoral populations," Letters in Biomathematics, vol. 5, no. 2, pp. S160-S177, 2018.

[35] H. Dritschel, S. Waters, A. Roller, and H. Byrne, "A mathematical model of cytotoxic and helper T cell interactions in a tumour microenvironment," Letters in Biomathematics, vol. 5, no. 2, pp. S36-S68, 2018.

[36] Y. Dong, G. Huang, R. Miyazaki, and Y. Takeuchi, "Dynamics in a tumor immune system with time delays," Applied Mathematics and Computation, vol. 252, pp. 99-113, 2015.

[37] M. Yu, Y. Dong, and Y. Takeuchi, "Dual role of delay effects in a tumour-immune system," Journal of Biological Dynamics, vol. 11, no. 2, pp. 334-347, 2017.

[38] M. Yu, G. Huang, Y. Dong, and Y. Takeuchi, "Complicated dynamics of tumor-immune system interaction model with distributed time delay," Discrete \& Continuous Dynamical Systems-B, vol. 25, no. 7, pp. 2391-2406, 2020.

[39] G. Caravagna, A. d'Onofrio, P. Milazzo, and R. Barbuti, "Tumour suppression by immune system through stochastic oscillations," Journal of Theoretical Biology, vol. 265, no. 3, pp. 336-345, 2010.

[40] J. T. George and H. Levine, "Stochastic modeling of tumor progression and immune evasion," Journal of Theoretical Biology, vol. 458, pp. 148-155, 2018.

[41] H.-P. Ren, Y. Yang, M. S. Baptista, and C. Grebogi, "Tumour chemotherapy strategy based on impulse control theory," Philosophical Transactions of the Royal Society A: Mathematical, Physical and Engineering Sciences, vol. 375, no. 2088, p. 20160221, 2017.

[42] Y. Deng and M. Liu, "Analysis of a stochastic tumor-immune model with regime switching and impulsive perturbations," Applied Mathematical Modelling, vol. 78, pp. 482-504, 2020.

[43] G. E. Mahlbacher, K. C. Reihmer, and H. B. Frieboes, "Mathematical modeling of tumor-immune cell interactions," Journal of Theoretical Biology, vol. 469, pp. 47-60, 2019.

[44] J. K. Hale, Theory of Functional Differential Equations, Springer, New York, NY, USA, 1977.

[45] A. Dhooge, W. Govaerts, and Y. A. Kuznetsov, "Matcont," ACM SIGSAM Bulletin, vol. 38, no. 1, pp. 21-22, 2004.

[46] A. Facciabene, G. T. Motz, and G. Coukos, "T-regulatory cells: key players in tumor immune escape and angiogenesis," Cancer Research, vol. 72, no. 9, pp. 2162-2171, 2012.

[47] K. Shitara and H. Nishikawa, "Regulatory T cells: a potential target in cancer immunotherapy," Annals of the New York Academy of Sciences, vol. 1417, no. 1, pp. 104-115, 2018.

[48] T. Zhang, J. Wang, Y. Li, Z. Jiang, and X. Han, "Dynamics analysis of a delayed virus model with two different transmission methods and treatments," Advances in Difference Equations, vol. 1, p. 2020, 2020.

[49] H. Zhang and T. Zhang, "The stationary distribution of a microorganism flocculation model with stochastic perturbation," Applied Mathematics Letters, vol. 103, p. 106217, 2020.

[50] T. Zhang, N. Gao, N. Gao, T. Wang, H. Liu, and Z. Jiang, "Global dynamics of a model for treating microorganisms in sewage by periodically adding microbial flocculants," Mathematical Biosciences and Engineering, vol. 17, no. 1, pp. 179201, 2020 . 